FIAN/TD-15/97

ITEP/TH-63/97

NBI-HE-97-61

\title{
5d and 6d Supersymmetric Gauge Theories: Prepotentials from Integrable Systems
}

\author{
A.Marshakov 中 \\ Niels Bohr Institute, Blegdamsvej 17, 2100 Copenhagen, Denmark \\ and \\ Theory Department, P.N.Lebedev Physics Institute, Leninsky prospect 53, Moscow 117924, Russia \\ and ITEP, Moscow 117259, Russia 2 \\ and \\ A.Mironov P $^{2}$ \\ Theory Department, P.N.Lebedev Physics Institute, Leninsky prospect 53, Moscow 117924, Russia \\ and ITEP, Moscow 117259, Russia
}

\begin{abstract}
We discuss $5 d$ and $6 d$ supersymmetric gauge theories in the target-space with compactified directions and with the matter hypermultiplets in fundamental representations in the framework of integrable systems. In particular, we consider the prepotentials of these theories and derive explicit formulas for their perturbative parts.
\end{abstract}

\footnotetext{
${ }^{1}$ E-mail address: mars@lpi.ac.ru, andrei@heron.itep.ru, marshakov@nbivms.nbi.dk

${ }^{2}$ Permanent address

${ }^{3}$ E-mail address: mironov@lpi.ac.ru,mironov@itep.ru
} 


\section{Introduction}

In this paper we continue our discussion on the Coulomb branches of gauge theories, which admit an exact treatment in the low-energy sector [1] and are related to integrable systems [2]-[15]. These are the theories with eight real supercharges (in accordance with common terminology that means $\mathcal{N}=2$ SUSY in four dimensions and $\mathcal{N}=1$ SUSY in $D=5,6$ ) with only four noncompact directions to have non-trivial instantonic contribution and, therefore, a non-trivial prepotential. It means, in particular, that we consider $5 d$ theories with the target space of topology $R^{4} \times S^{1}$ and $6 d$ theories with the target space of topology $R^{4} \times T^{2}$. Below we give a detailed analysis of the results for the prepotentials of these higher dimensional theories. This paper continues our investigation of the prepotentials started in [10] and, in particular, gives some support to the integrable structures of higher dimensional theories proposed in [14, 15.

We consider here the SUSY theories with massive hypermultiplets in fundamental representations. Therefore, in accordance with [5, 6, 15], the corresponding integrable structures are given by (classical) spin chains: the $X X Z$ chain in $5 d$ and $X Y Z$ (Sklyanin) chain in $6 d$. Below we concentrate on the issues related to the prepotentials and associated analytic structures on Riemann surfaces: differentials, their periods etc. In particular, we calculate the perturbative prepotentials which coincide with the expected from general considerations of quantum field theory. It gives some support to the identification of the integrable spin-chains with the SUSY QCD in higher dimensions.

An integrable system, i.e. a spectral curve and a generating 1-differential $d S$ [16], allows to define the prepotential in the framework of the Seiberg-Witten (SW) anzatz [1], where it can be identified with the (logarithm of) tau-function of the Whitham hierarchy [2]. In sect.2 we remind this procedure for the standard case of $4 d \mathcal{N}=2$ QCD. In sect.3 we extend it to the $5 d$ case, in particular, we advocate there the most effective method of calculating the prepotentials based on residue formulas first studied in the framework of the SW theory in connection with the associativity equations [9, 10]. The procedure becomes especially explicit when computing the perturbative prepotentials. In sect.4 we discuss $6 d$ theories described by the $X Y Z$ spin chain and sect.5 contains some discussion. Appendix contains some definitions and formulas on elliptic functions we use throughout sect. 4 .

\section{$24 d$ supersymmetric theories: the Seiberg-Witten anzatz}

First, as a warm-up example we consider the integrable system corresponding to the $4 d$ SUSY QCD and explain how the data obtained from integrable theory can be associated with the objects in nonperturbative SUSY theories, in the context of the Seiberg-Witten anzatz [1]. Our presentation

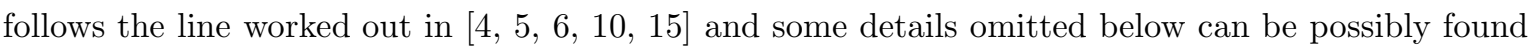
there.

\section{$2.1 \quad 4 d$ theories and $X X X$ chain}

Let us remind, first, the definition of the $s l(2)$ (integrable) $X X X$ spin chain. The most convenient formulation is based on the $2 \times 2$ Lax matrix (see for example [17]), which for the $\operatorname{sl}(2) X X X$ chain is

$$
L(\lambda)=\lambda \cdot \mathbf{1}+\sum_{a=1}^{3} S_{a} \cdot \sigma^{a} .
$$


and the Poisson brackets on the space of the dynamical variables $S_{a}, a=1,2,3$ in the $2 \times 2$ formalism are implied by quadratic r-matrix relations [18]

$$
\left\{L(\lambda) \stackrel{\otimes}{,} L\left(\lambda^{\prime}\right)\right\}=\left[r\left(\lambda-\lambda^{\prime}\right), L(\lambda) \otimes L\left(\lambda^{\prime}\right)\right],
$$

with the rational $r$-matrix

$$
r(\lambda)=\frac{1}{\lambda} \sum_{a=1}^{3} \sigma^{a} \otimes \sigma^{a} .
$$

Following standard procedure [0], one should consider the chain of the Lax operators (2.1) associated to each site of the chain and commuting with each other at different sites. The linear problem in the spin chain 17] can be defined by

$$
L_{i}(\lambda) \Psi_{i}(\lambda)=\Psi_{i+1}(\lambda)
$$

where $\Psi_{i}(\lambda)$ is the two-component Baker-Akhiezer function. One can introduce the transfer matrix acting from $i$-th site to $i+n$-th taking product of the Lax matrices (2.5). The crucial property of the relation (2.2) is that it is multiplicative, i.e. any transfer matrix, being product of the form

$$
\begin{gathered}
T_{n}(\lambda) \equiv L_{n}(\lambda) \ldots L_{1}(\lambda) \\
T_{n}(\lambda) \Psi_{i}(\lambda)=\Psi_{i+n}(\lambda)
\end{gathered}
$$

satisfies the same relation

$$
\left\{T_{n}(\lambda) \stackrel{\otimes}{,} T_{n}\left(\lambda^{\prime}\right)\right\}=\left[r\left(\lambda-\lambda^{\prime}\right), T_{n}(\lambda) \otimes T_{n}\left(\lambda^{\prime}\right)\right],
$$

provided all $L_{i}$ in product (2.5) are independent, $\left\{L_{i}, L_{j}\right\}=0$ for $i \neq j$. The relations (2.2) are also true for the inhomogeneous $T$-matrix $\left(\lambda \rightarrow \lambda+\lambda_{i}\right.$ at $i$-th site; in the applications to $\mathcal{N}=2$ SUSY gauge theories the chain has to be taken of the length $N_{c}$ [5])

$$
T_{N_{c}}(\lambda)=\prod_{N_{c} \geq i \geq 1}^{\curvearrowleft} L_{i}\left(\lambda-\lambda_{i}\right)
$$

still satisfying (2.6) with the same $r$-matrix (2.3).

In the $\operatorname{sl}(2) X X X$ case the $r$-matrix relations 2.2) are equivalent just to the common $s l(2)$ commutation relation (written in terms of the Poisson brackets)

$$
\left\{S_{a}, S_{b}\right\}=i \epsilon_{a b c} S_{c}
$$

i.e. $\left\{S_{a}\right\}$ plays the role of angular momentum ("classical spin") variables giving the name "spin-chains" to the whole class of systems. The algebra 2.8) has an obvious Casimir operator (an invariant, which Poisson commutes with all the generators $S_{a}$ ),

$$
K^{2}=\sum_{a=1}^{3} S_{a} S_{a},
$$

so that

$$
\begin{gathered}
\operatorname{det}_{2 \times 2} L(\lambda)=\lambda^{2}-K^{2}, \\
\operatorname{det}_{2 \times 2} T_{N_{c}}(\lambda)=\prod_{i=1}^{N_{c}} \operatorname{det}_{2 \times 2} L_{i}\left(\lambda-\lambda_{i}\right)=\prod_{i=1}^{N_{c}}\left(\left(\lambda-\lambda_{i}\right)^{2}-K_{i}^{2}\right)= \\
=\prod_{i=1}^{N_{c}}\left(\lambda-m_{i}^{+}\right)\left(\lambda-m_{i}^{-}\right)=Q_{2 N_{c}}(\lambda),
\end{gathered}
$$


where we assumed that the values of spin $K$ can be different at different nodes of the chain, and

$$
m_{i}^{ \pm}=\lambda_{i} \pm K_{i}
$$

While the determinant of monodromy matrix 2.10) depends on dynamical variables only through the Casimir functions $K_{i}$ of the Poisson algebra, the dependence of

$$
P_{N_{c}}(\lambda)=\frac{1}{2} \operatorname{Tr}_{2 \times 2} T_{N_{c}}(\lambda)=\prod_{i=1}^{N_{c}}\left(\lambda-\lambda_{i}\right)
$$

upon dynamical variables is less trivial. Still, as usual for integrable systems

$$
\left\{P_{N_{c}}(\lambda), P_{N_{c}}\left(\lambda^{\prime}\right)\right\}=\frac{1}{4}\left\{\operatorname{Tr} T_{N_{c}}(\lambda), \operatorname{Tr} T_{N_{c}}\left(\lambda^{\prime}\right)\right\}=\frac{1}{4} \operatorname{Tr}\left[r\left(\lambda-\lambda^{\prime}\right), T_{N_{c}}(\lambda) \otimes T_{N_{c}}\left(\lambda^{\prime}\right)\right]=0
$$

i.e. the polynomial $P_{N_{c}}(\lambda)$ (2.12) depends on $S_{a}^{(i)}$ only through the Hamiltonians of spin chain (Poisson-commuting with each other).

The (quasi)periodic boundary conditions imposed to the Baker-Akhiezer function

$$
\Psi_{i+N_{c}}(\lambda)=w \Psi_{i}(\lambda)
$$

give rise to the spectral curve equation for the periodic spin chain

$$
\operatorname{det}\left(T_{N_{c}}(\lambda)-w\right)=0
$$

In the particular case of $s l(2) X X X$ spin chains, the spectral equation acquires the form 1

$$
w+\frac{Q_{N_{f}}(\lambda)}{w}=2 P_{N_{c}}(\lambda)
$$

where the number of matter multiplets $N_{f} \leq 2 N_{c}$ depending on a particular "degeneracy" of the full chain and, as follows from (2.1), (2.12) $\sum \lambda_{i}=0$.

It is relatively easy to get the exact expressions for the Hamiltonians (the explicit examples of monodromy matrices and Hamiltonians for $N_{c}=2$ and 3 can be found in [5]). The Hamiltonians depend non-trivially on the $\lambda_{i}$-parameters (inhomogeneities of the chain) and the coefficients in the spectral equation (2.15) depend only on the Hamiltonians and symmetric functions of the massparameters (2.11), i.e. the dependence of $\left\{\lambda_{i}\right\}$ and $\left\{K_{i}\right\}$ is rather special. This property is crucial for identification of the $m$-parameters with the masses of the matter supermultiplets in the $\mathcal{N}=2$ SQCD.

\subsection{Spectral curves and prepotentials}

Let us turn now to the $4 d \mathrm{SW}$ anzatz [1], which can be formulated in the following way. The $\mathcal{N}=2$ SUSY vector multiplet has necessarily (complex) scalars with the potential $V(\phi)=\operatorname{Tr}\left[\phi, \phi^{\dagger}\right]^{2}$ whose minima (after factorization over the gauge group) correspond to the diagonal $\left(\left[\phi, \phi^{\dagger}\right]=0\right)$, constant and (in the theory with $S U\left(N_{c}\right)$ gauge group) traceless matrices. Their invariants

$$
\operatorname{det}(\lambda-\phi) \equiv P_{N_{c}}(\lambda)=\sum_{k=0}^{N_{c}} s_{N_{c}-k} \lambda^{k}
$$

\footnotetext{
${ }^{1}$ Eq. 2.11) implies that the limit of vanishing masses, all $m_{i}^{ \pm}=0$, is associated with the homogeneous chain (all $\left.\lambda_{i}=0\right)$ and vanishing spins at each site (all $\left.K_{i}=0\right)$.

${ }^{2}$ In general case of $\operatorname{sl}(p)$ chains the spectral equation is of the $p$-th order in $w$, see 5,15$]$ and below.
} 
(the total number of algebraically independent ones is rank $S U\left(N_{c}\right)=N_{c}-1$ ) parametrize the moduli space of the (Coulomb branch of the) theory. Due to the Higgs effect the off-diagonal part of the gauge field $\mathbf{A}_{\mu}$ becomes massive, since

$$
\left[\boldsymbol{\phi}, \mathbf{A}_{\mu}\right]_{i j}=\left(\phi_{i}-\phi_{j}\right) \mathbf{A}_{\mu}^{i j}
$$

while the diagonal part, as it follows from (2.18) remains massless, i.e. the gauge group $G=S U\left(N_{c}\right)$ breaks down to $U(1)^{\operatorname{rank} G}=U(1)^{N_{c}-1}$.

The effective abelian $U(1)^{N_{c}-1}$ theory is formulated in terms of a (finite-dimensional) integrable system: the spectral curve $\Sigma$ defined over the genus-dimensional subspace of the full moduli space, e.g.

$$
\mathcal{P}(\lambda, w)=2 P_{N_{c}}(\lambda)-w-\frac{1}{w}=0
$$

for the pure $S U\left(N_{c}\right)$ gauge theory and the generating differential

$$
d S=\lambda \frac{d w}{w}
$$

whose basic property is that its derivatives over $N_{c}-1$ moduli give rise to holomorphic differentials. From the point of view of integrable system, the spectral curve express the condition of the common spectrum of two operators, usually the Lax operator $\mathcal{L}$ and some auxiliary operator, which was taken above to be the shift operator $T_{N_{c}}$ (2.5). From practical point of view one can work in the basis of eigenvalues of one of the operators: in the formalism presented above we have chosen the basis of the Lax eigenfunctions - the wave functions which explicitly depend on the Lax eigenvalue $\lambda$, then another operator acts in this basis as a $\lambda$-dependent matrix. As a result, the full spectral curve $\Sigma$ arises as a ramified covering over some bare spectral curve:

$$
\mathcal{P}(\lambda ; z)=0
$$

where $\lambda$ or $z\left(z=\log w\right.$ in (2.19) ) can be considered as a co-ordinate on bare spectral curve $\Sigma_{0}$. In the case of the gauge group $G=S U\left(N_{c}\right)$, the function $\mathcal{P}$, caused by (2.17), is a polynomial of degree $N_{c}$ in $\lambda$. If one adds the fundamental matter hypermultiplets 19 one should also complete the set of moduli by their masses $m_{\alpha}\left(\alpha=1, \ldots, N_{f}\right)$ and modify the form of the curve (2.19) to (2.16). Then, natural generating differential is

$$
d S=\lambda \frac{d w}{w}
$$

There is, however, another generating differential usually choosen in literature [1, 5, 19], which is expressed through the variable $W \equiv \frac{w}{\sqrt{Q_{N_{f}}(\lambda)}}$ :

$$
d S=\lambda \frac{d W}{W}
$$

This variable is naturally associated with the curve written in some more symmetric form

$$
W+\frac{1}{W}=\frac{2 P_{N_{c}}(\lambda)}{\sqrt{Q_{N_{f}}(\lambda)}}
$$

The both choices of the differentials lead to the same results [10]. In this paper, we use the differential (2.24) as better suited for perturbative calculations.

\footnotetext{
${ }^{3}$ In terms of brane picture it depends on the exact form of embedding of a spectral curve into (eleven-dimensional) target space (see, for example, 12, 14, 15, 20] for more detailed discussion of this point).
} 
The coefficients $s_{I}$ of the function $\mathcal{P}$ parametrize the (subspace of) moduli space of complex structures $\mathcal{M}_{\Sigma}$ of the curve $\Sigma$. The Hamiltonians or action variables (integrals of motion) are specific co-ordinates on moduli space. From the four-dimensional point of view, the co-ordinates $s_{I}$ include $s_{i}$ - (the Schur polynomials of) the adjoint-scalar expectation values $h_{k}=\frac{1}{k}\left\langle\operatorname{Tr} \phi^{k}\right\rangle$ of the vector $\mathcal{N}=2$ supermultiplet, as well as $s_{\alpha}=m_{\alpha}$ - the masses of the hypermultiplets. One associates with each handle of $\Sigma$ a gauge modulus and with each puncture - a massive hypermultiplet with mass given by residue of $d S$ in the puncture.

The generating 1-form $d S \cong \lambda d z$ is meromorphic on $\Sigma$ (hereafter the equality modulo total deriva-

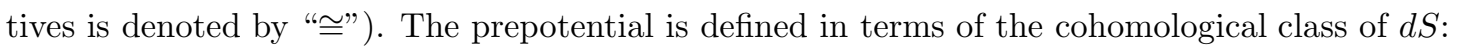

$$
\begin{gathered}
a_{I}=\oint_{A_{I}} d S, \quad \frac{\partial \mathcal{F}}{\partial a_{I}}=\int_{B_{I}} d S \\
A_{I} \circ B_{J}=\delta_{I J} .
\end{gathered}
$$

The cycles $A_{I}$ include the $A_{i}$ 's wrapping around the handles of $\Sigma$ and $A_{\alpha}$ 's, going around the singularities of $d S$. The conjugate contours $B_{I}$ include the cycles $B_{i}$ and the non-closed contours $B_{\alpha}$, ending at the singularities of $d S$ (see [10] for details). The integrals $\int_{B_{\alpha}} d S$ are actually divergent, but the coefficient of divergent part is equal to residue of $d S$ at particular singularity, i.e. to $a_{\alpha}$. Thus, the divergent contribution to the prepotential is quadratic in $a_{\alpha}$, while the prepotential is normally defined modulo quadratic combination of its arguments (which just fixes the bare coupling constant). In particular models $\oint_{A, B} d S$ for some pairs of conjugate contours are identically zero on entire $\mathcal{M}$ : such pairs are not included into the set of indices $\{I\}$.

Note that the data $(\Sigma, d S)$ with such properties are exactly the definition of the integrable system in the sense of [16] (see also [7] and references therein for details). The period matrix of $\Sigma: T_{i j}\left(a_{i}\right)=$ $\frac{\partial^{2} \mathcal{F}}{\partial a_{i} \partial a_{i}}$ as a function of the action variables $a_{i}$ gives the set of coupling constants in the effective abelian $U(1)^{N_{c}-1}$ theory while action variables themselves are identified with the masses of the BPS states $M^{2} \sim\left|\mathbf{n a}+\mathbf{m a} \mathbf{a}_{D}\right|^{2}$ with the $(\mathbf{n}, \mathbf{m})$ "electric" and "magnetic" charges. As an output of all these definitions, one can derive the following residue formula [9, 10] for the derivatives of prepotential

$$
\frac{\partial^{3} \mathcal{F}}{\partial a_{I} \partial a_{J} \partial a_{K}}=\frac{\partial T_{I J}}{\partial a_{K}}=\underset{d z=0}{\operatorname{res}} \frac{d \omega_{I} d \omega_{J} d \omega_{K}}{d z d \lambda}
$$

where $d \omega_{I}$ are the canonical holomorphic 1-differentials satisfying $\oint_{A_{I}} d \omega_{J}=\delta_{I J}$. Their basic property $\oint_{B_{I}} d \omega_{J}=T_{I J}$ can be obtained by differentiating the meromorphic differential $d S$ w.r.t. to the moduli $a_{I}$.

This formula can be effectively used for the calculations of the prepotentials term by term, in particular, for the perturbative calculations.

\subsection{Perturbative prepotential}

Now, following [10] we discuss the simplest case of degenerate curves corresponding to the perturbative formulas. In the perturbative limit $\left(\frac{\Lambda_{Q C D}}{\langle\phi\rangle} \rightarrow 0\right)$ the second term in 2.24) vanishes, the curve acquires the form

$$
W=2 \frac{P_{N_{c}}(\lambda)}{\sqrt{Q_{N_{f}}(\lambda)}}
$$


(a rational curve with punctures 4 ) and the generating differential (2.23) turns into

$$
d S_{\text {pert }}^{(4)}=\lambda d \log \left(\frac{P_{N_{c}}(\lambda)}{\sqrt{Q_{N_{f}}(\lambda)}}\right)
$$

We remind, first, the simplest case of the pure gauge $S U\left(N_{c}\right)$ Yang-Mills theory (2.19), (2.20), i.e. $Q(\lambda)=1$. Now the set of the $N_{c}-1$ independent canonical holomorphic differentials is

$$
d \omega_{i}=\left(\frac{1}{\lambda-\lambda_{i}}-\frac{1}{\lambda-\lambda_{N_{c}}}\right) d \lambda=\frac{\lambda_{i N_{c}} d \lambda}{\left(\lambda-\lambda_{i}\right)\left(\lambda-\lambda_{N_{c}}\right)}, \quad i=1, \ldots, N_{c}-1, \quad \lambda_{i j} \equiv \lambda_{i}-\lambda_{j}
$$

and the $A$-periods $a_{i}=\lambda_{i}$ (the independent ones are, say, with $i=1, \ldots, N_{c}-1$ ) coincide with the roots of polynomial $P_{N_{c}}(\lambda)$ (2.12). The prepotential can be now computed via residue formulas [10]

$$
\mathcal{F}_{i j k}=\underset{d \log P_{N_{c}}=0}{\operatorname{res}} \frac{d \omega_{i} d \omega_{j} d \omega_{k}}{d \log P_{N_{c}}(\lambda) d \lambda}
$$

which can be explicitly calculated. The only technical trick is that it is easier to compute the residues at the poles of $d \omega$ 's instead of zeroes of $d \log P$. This can be done immediately since there are no contributions from the infinity $\lambda=\infty$. The final results have the following form

$$
\begin{gathered}
\mathcal{F}_{i i i}=\sum_{k \neq i} \frac{1}{\lambda_{i k}}+\frac{6}{\lambda_{i N_{c}}}+\sum_{k \neq N_{c}} \frac{1}{\lambda_{k N_{c}}}, \\
\mathcal{F}_{i i j}=\frac{3}{\lambda_{i N_{c}}}+\frac{2}{\lambda_{j N_{c}}}+\sum_{k \neq i, j, N_{c}} \frac{1}{\lambda_{k N_{c}}}-\frac{\lambda_{j N_{c}}}{\lambda_{i N_{c}} \lambda_{i j}}, \quad i \neq j, \\
\mathcal{F}_{i j k}=2 \sum_{l \neq N_{c}} \frac{1}{\lambda_{l N_{c}}}-\sum_{l \neq i, j, k, N_{c}} \frac{1}{\lambda_{l N_{c}}}, \quad i \neq j \neq k ;
\end{gathered}
$$

giving rise to the prepotential formula

$$
\begin{gathered}
\mathcal{F}=\frac{1}{4} \sum_{i j} f^{(4)}\left(\lambda_{i j}\right) \\
f^{(4)}(x)=x^{2} \log x^{2}
\end{gathered}
$$

Now let us turn back to the case of $4 d \mathcal{N}=2$ QCD with massive hypermultiplets Then, there arise additional differentials corresponding to the derivatives of $d S(2.28)$ with respect to masses. They are of the form

$$
d \omega_{\alpha}=-\frac{1}{2} \frac{d \lambda}{\lambda-\lambda_{\alpha}}
$$

It is again straightforward to use the residue formula

$$
\mathcal{F}_{I J K}=\underset{d \log \frac{P}{\sqrt{Q}}=0}{\operatorname{res}} \frac{d \omega_{I} d \omega_{J} d \omega_{K}}{d \log \frac{P(\lambda)}{\sqrt{Q(\lambda)}} d \lambda}, \quad\{I, J, K, \ldots\}=\{i, j, k, \ldots \mid \alpha, \beta, \gamma, \ldots\}
$$

\footnotetext{
${ }^{4}$ These punctures emerge as a degeneration of the handles of the hyperelliptic surface so that the $A$-cycles encircle the punctures.

${ }^{5}$ Let us note that, despite the differentials $d \omega_{\alpha}$ have the pole at infinity, this does not contribute into the residue formula because of the quadratic pole of $d \lambda$ in the denominator.
} 
and obtain

$$
\begin{gathered}
\mathcal{F}_{i i i}=\sum_{k \neq i} \frac{1}{\lambda_{i k}}+\frac{6}{\lambda_{i N_{c}}}+\sum_{k \neq N_{c}} \frac{1}{\lambda_{k N_{c}}}-\frac{1}{2} \sum_{\alpha} \frac{\lambda_{i N_{c}}}{\lambda_{i \alpha} \lambda_{\alpha N_{c}}}, \\
\mathcal{F}_{i i j}=\frac{3}{\lambda_{i N_{c}}}+\frac{2}{\lambda_{j N_{c}}}+\sum_{k \neq i, j, N_{c}} \frac{1}{\lambda_{k N_{c}}}-\frac{\lambda_{j N_{c}}}{\lambda_{i N_{c}} \lambda_{i j}}-\frac{1}{2} \sum_{\alpha} \frac{1}{\lambda_{\alpha N_{c}}}, \quad i \neq j, \\
\sum_{l \neq N_{c}} \frac{1}{\lambda_{l N_{c}}}-\sum_{l \neq i, j, k, N_{c}} \frac{1}{\lambda_{l N_{c}}}-\frac{1}{2} \sum_{\alpha} \frac{1}{\lambda_{\alpha N_{c}}}, \quad i \neq j \neq k ; \\
\mathcal{F}_{i i \alpha}=\frac{1}{2}\left(\frac{1}{\lambda_{i \alpha}}-\frac{1}{\lambda_{\alpha N_{c}}}\right) ; \\
\mathcal{F}_{i j \alpha}=-\frac{1}{2} \frac{1}{\lambda_{\alpha N_{c}}}, \quad i \neq j ; \\
\mathcal{F}_{i \alpha \alpha}=-\frac{1}{2} \frac{\lambda_{i N_{c}}}{\lambda_{i \alpha} \lambda_{\alpha N_{c}}} ; \\
\mathcal{F}_{\alpha \alpha \beta}=-\frac{1}{2} \sum_{i} \frac{1}{\lambda_{\alpha i}}+\frac{1}{4} \sum_{\beta} \frac{1}{\lambda_{\alpha \beta}} ; \quad \alpha \neq \beta ; \\
\mathcal{F}_{i \alpha \beta}=\mathcal{F}_{\alpha \beta \gamma}=0
\end{gathered}
$$

These formulas immediately lead to the prepotential

$$
\mathcal{F}=\frac{1}{4} \sum_{i, j} f^{(4)}\left(a_{i j}\right)-\frac{1}{4} \sum_{i, \alpha} f^{(4)}\left(a_{i}-m_{\alpha}\right)+\frac{1}{16} \sum_{\alpha, \beta} f^{(4)}\left(m_{\alpha}-m_{\beta}\right)
$$

upon the identification $\lambda_{i}=a_{i}$ and $\lambda_{\alpha}=m_{\alpha}$.

\section{$3 \quad X X Z$ spin chain and $5 d$ theories}

Now we are going to describe the generic integrable system behind the $5 d$ theories - the inhomogeneous $X X Z$ spin chain, and reproduce from the integrable system the main ingredients of the $5 d \mathrm{SW}$ theory: the spectral curve of and generating 1-differential $d S$. As in $4 d$ case it allows to construct explicitly the holomorphic differentials on spectral curve and calculate the perturbative part of $5 d$ prepotential.

\section{$3.1 \quad X X Z$ spin chain}

In $2 \times 2$ formalism the generalization of $4 d$ formulas is straightforward: the Lax matrix for the $X X Z$ $(s l(2))$ spin magnetic is defined on a cylinder and has the form 17]

$$
L(\mu)=\left(\begin{array}{cc}
\mu e^{S_{0}}-\mu^{-1} e^{-S_{0}} & 2 S_{-} \\
2 S_{+} & \mu e^{-S_{0}}-\mu^{-1} e^{S_{0}}
\end{array}\right)
$$

In fact, from the point of view of integrable systems more natural spectral parameter is $\xi=\log \mu$ so that the matrix elements of the Lax operator (3.1) are trigonometric functions of $\xi$. The Poisson bracket of the Lax operator (3.1)

$$
\left\{L(\mu) \stackrel{\otimes}{,} L\left(\mu^{\prime}\right)\right\}=\left[r\left(\mu-\mu^{\prime}\right), L(\mu) \otimes L\left(\mu^{\prime}\right)\right]
$$


is defined by the trigonometric $r$-matrix

$$
r(\xi)=\frac{i}{\sinh \pi \xi}\left(\sigma_{1} \otimes \sigma_{1}+\sigma_{2} \otimes \sigma_{2}+\cosh \pi \xi \sigma_{3} \otimes \sigma_{3}\right)
$$

so that (3.2) gives rise to the following Poisson algebra of $S_{i}$ 's:

$$
\left\{S_{+}, S_{0}\right\}= \pm S_{ \pm} ; \quad\left\{S_{+}, S_{-}\right\}=\sinh 2 S_{0}
$$

The second Casimir function is now

$$
K=\cosh 2 S_{0}+2 S_{+} S_{-}
$$

The non-linear relations (3.4) remind the commutation relations of quantum algebra $U_{q}(s l(2))$, this is a generalization the fact that the $X X X$ Poisson brackets (2.8) reproduce the classical algebra sl(2). The value of deformation parameter $q$ is inessential in (3.4) and can be put equal to unity; in fact, it is proportional to the radius of the target space circle $R_{5}$ in corresponding $5 d$ SUSY theory and can be easily restored replacing each generator $S_{i}$ by $R_{5} S_{i}$. Hereafter, we omit $R_{5}$ from all the formulas except for some special cases below.

Again as in $4 d$ case one should consider the chain with $N_{c}$ sites, now with the Lax operators (3.1) associated to each site

$$
L_{i}(\mu) \Psi_{i}(\mu)=\Psi_{i+1}(\mu)
$$

and commuting with each other for $i \neq j$; introduce the inhomogeneities $\xi_{i}$ which depend on the site of chain replacing $\xi \rightarrow \xi-\xi_{i}$ and impose periodic boundary conditions. As before the transfer matrix acting from $i$-th to $i+N_{c}$-th site is the product of the Lax matrices 2.5)

$$
T_{N_{c}}(\mu)=\prod_{N_{c} \geq i \geq 1}^{\curvearrowleft} L_{i}\left(\frac{\mu}{\mu_{i}}\right)
$$

and the (quasi)periodic boundary conditions

$$
\Psi_{i+N_{c}}(\mu)=-w \Psi_{i}(\mu)
$$

provide the spectral curve equation now for the $X X Z$ spin chain

$$
\operatorname{det}\left(T_{N_{c}}(\mu)+w \cdot \mathbf{1}\right)=0
$$

The coefficients in this equation can be taken as a complete set of integrals of motion.

The manifest form of the curve in the $X X Z$ case can be derived using explicit expression for the Lax matrix (3.1) and reads

$$
w+\frac{Q_{2 N_{c}}\left(e^{2 \xi}\right)}{e^{2 N_{c} \xi+2 \sum_{i} \xi_{i}} w}=2 e^{-N_{c} \xi-\sum_{i} \xi_{i}} P_{N_{c}}\left(e^{2 \xi}\right)=2 e^{-N_{c} \xi-\sum_{i} \xi_{i}}\left(e^{2 N_{c} \xi}+\ldots+e^{2 \sum \xi_{i}}\right)
$$

Changing the variables $e^{2 \xi}=\mu^{2} \equiv \lambda, w \rightarrow e^{-N_{c} \xi-\sum_{i} \xi_{i}} w$, this curve can be rewritten in the hyperelliptic form in $\lambda$ variables:

$$
w+\frac{Q_{2 N_{c}}(\lambda)}{w}=2 P_{N_{c}}(\lambda), \quad Y^{2}=P_{N_{c}}^{2}(\lambda)-Q_{2 N_{c}}(\lambda), \quad Y \equiv \frac{1}{2}\left(w-\frac{Q_{2 N_{c}}(\lambda)}{w}\right)
$$

\footnotetext{
${ }^{6} \mathrm{In}$ fact, the coefficient in front of $P_{N_{c}}$, instead of 2 , is equal to $2 \cosh \prod_{i}^{N_{c}} e^{S_{0, i}}$. It is, however, the integral of motion that can be put equal to any number. We always fix $\sum_{i}^{N_{c}} S_{0, i}$ to be zero.
} 
while in terms of "true" spectral parameter $\xi$ this curve looks considerably more tricky. However, one can work with variable $\lambda$ taking into account that it lives on a cylinder or sphere with two marked points. In the equation (3.11), the polynomial

$$
Q_{2 N_{c}}(\lambda)=\prod_{i}\left(\lambda^{2}-2 K_{i} e^{2 \xi_{i}} \lambda+e^{4 \xi_{i}}\right) \equiv \prod_{i}\left(\lambda-e^{2 m_{i}^{(+)}}\right)\left(\lambda-e^{2 m_{i}^{(-)}}\right)
$$

defines the masses $m_{i}^{( \pm)}$of the matter hypermultiplets. They turn out to be functions of the second Casimirs $K_{i}$ of the algebra (3.4) at $i$-th site and corresponding inhomogeneity to be compared with (2.11)

$$
m_{i}^{( \pm)}=\xi_{i} \pm \frac{1}{2} \log \left(K_{i}+\sqrt{K_{i}^{2}-1}\right)
$$

At the same time, the second polynomial $P_{N_{c}}(\lambda)$ depends on the integrals of motion (mutually Poissoncommuting with each other) playing the role of moduli of gauge theory in the framework of the SW theory. Modulo these subtleties, the curve (3.11) is very similar to the curves arising for $4 d$ theories. However, the difference coming from different generating 1-differentials $d S$ turns to be very crucial (see, for example, the discussion of residue formula and perturbative prepotentials).

It is easy to compare the curves (3.11) with those of [22], rewriting them in the form

$$
w+\frac{\prod_{\alpha} \sinh \left(\xi-m_{\alpha}\right)}{w}=2 \prod_{i} \sinh \left(\xi-a_{i}\right)
$$

where we rescaled $w \rightarrow e^{N_{c} \xi+\sum_{i} \xi_{i}} w$, denoted the roots of the polynomial $P_{N_{c}}(\lambda)$ (3.10) $\lambda_{i} \equiv e^{2 a_{i}}$ and made use of formula (3.13) and the manifest form of the leading and the constant terms in this polynomial. Comparing (3.14) with (3.10), one finds, in particular, that

$$
\sum a_{i}=\sum \xi_{i}=\frac{1}{2} \sum m_{\alpha}
$$

The condition (3.15) is not, however, absolutely necessary in the context of $X X Z$ chains and $5 d$ theories (we return to this point later in s.3.3). It emerges only in the standard $X X Z$ chain. In the context of SW theory one needs rather to consider the twisted $X X Z$ model [21, 15] ?. It is characterized by the Lax operator

$$
L_{i}(\mu)=\left(\begin{array}{cc}
\mu e^{S_{0, i}-\xi_{i}}-\alpha_{i} \mu^{-1} e^{-S_{0, i}+\xi_{i}} & 2 S_{-, i} \\
2 S_{+, i} & \mu e^{-S_{0, i}-\xi_{i}}-\beta_{i} \mu^{-1} e^{S_{0, i}+\xi_{i}}
\end{array}\right)
$$

with generally non-unit constants $\alpha_{i}, \beta_{i}$. These constants provide arbitrary coefficient in front of product in the r.h.s. of (3.14) (which can be also regulated by the integral of motion $\sum_{i}^{N_{c}} S_{0, i}$ ) and, thus, break the condition (3.15). They are also important for careful treatment of dimensional transmutation (i.e. decreasing the number of massive hypermultiplets) [15]. The maximally degenerated case is related to the pure gauge theory when all the masses become infinite. In this case, one gets the relativistic Toda chain, first discussed in this context in [8].

There certainly exist all intermediate degenerations when all but $N_{f}$ masses are brought to infinity. This system, corresponding to the gauge theory with $N_{f}<2 N_{c}$ massive hypermultiplets, is described by the chain with some of the sites degenerated and some of them not. The corresponding spectral curve has the form

$$
w+\frac{Q_{N_{f}}(\lambda)}{w}=2 P_{N_{c}}(\lambda), \quad Q_{N_{f}}(\lambda)=\prod_{\alpha}^{N_{f}}\left(\lambda-e^{2 m_{\alpha}}\right), \quad P_{N_{c}}(\lambda)=\prod_{i}^{N_{c}}\left(\lambda-e^{2 a_{i}}\right)
$$

\footnotetext{
${ }^{7}$ We are grateful to S.Kharchev for the discussion of the twisted spin chains.
} 
or

$$
w+\frac{\prod_{\alpha}^{N_{f}} \sinh \left(\xi-m_{\alpha}\right)}{w}=2 e^{\left(N_{c}-N_{f} / 2\right) \xi+\sum a_{i}-1 / 2 \sum m_{\alpha}} \prod_{i}^{N_{c}} \sinh \left(\xi-a_{i}\right)
$$

(where we again rescaled $w \rightarrow e^{\frac{1}{2}\left(N_{f} \xi+\sum m_{\alpha}\right)} w$; this curve also coincides with that in [22]). Below we mainly discuss the generic degenerated system with $N_{f}$ not necessarily equal to $2 N_{c}$. As we already noted, the particular coefficient $e^{\sum a_{i}-1 / 2 \sum m_{\alpha}}$ can be ,in principle, replaced by any other number, say put equal to unity, which literally corresponds to [22]. It does not influence the result and we will discuss the reason below.

Note that the form (3.14) of the spectral curve is perfectly designed for taking the $4 d$ limit. Indeed, one can restore $R_{5}$-dependence in this formula multiplying each $\xi_{i}$ and mass parameter by $R_{5}$. In terms of algebra (3.4) it means that each generator has to be multiplied by $R_{5}$ and so does the spectral parameter. Then, one immediately reproduces the results of sect.4.

\subsection{Relativistic integrable systems and $5 d$ theories}

The general scheme of the SW anzatz presented above in the $4 d$ case can be almost literally transferred to the "relativistic" $5 d \mathcal{N}=2$ SUSY gauge models with one compactified dimension. One can understand the reason of this "relativization" in the following way. Considering four plus one compact dimensional theory one should take into account the contribution of all Kaluza-Klein modes to each 4-dimensional field. Roughly speaking it leads to the 1-loop contributions to the effective charge of the form

$$
T_{i j} \sim \sum_{\text {masses }} \log \text { masses } \sim \sum_{m} \log \left(a_{i j}+\frac{m}{R_{5}}\right) \sim \log \prod_{m}\left(R_{5} a_{i j}+m\right) \sim \log \sinh R_{5} a_{i j}
$$

i.e. coming from $4 d$ to $5 d$ one should make a substitution $a_{i j} \rightarrow \sinh R_{5} a_{i j}$, at least, in the formulas for the perturbative prepotential. The similar change of variables corresponds to relativization of integrable systems, which implies a sort of "Lie group generalization" of the "ordinary" integrable systems related to Lie algebras. In terms of $2 \times 2$ Lax representation, the "relativization" corresponds to replacing by trigonometric $r$-matrices and $L$-operators the rational ones: in algebraic terms this corresponds to turning from Yangians to quantum affine algebras. In particular, it means that now it is natural to consider both parameters $\xi=\frac{1}{2} \log \lambda$ and $z=\log W$ as co-ordinates on cylinder, i.e. both bare spectral curves become the cylinders.

Similar arguments imply that, instead of differential $(2.20) d S^{(4)}=\lambda d \log W$, one has now to consider the differential 8,10$]$

$$
d S^{(5)}=\xi \frac{d W}{W}=\frac{1}{2} \log \lambda \frac{d W}{W}
$$

so that, despite the similarities between the $5 d$ and $4 d$ spectral curves, the periods of $d S^{(5)}$ are different from the periods of $d S^{(4)}$.

The difference with the $4 d$ case results also in a slightly different residue formula. Indeed, repeating the derivation of the paper $[10]$, one can see that

$$
\frac{\partial^{3} \mathcal{F}}{\partial a_{I} \partial a_{J} \partial a_{K}}=2 \underset{\substack{d z=0 \\ \lambda=0}}{\operatorname{res}} \frac{d \omega_{I} d \omega_{J} d \omega_{K}}{d z \frac{d \lambda}{\lambda}}
$$

The crucial distinction is that the contour in (3.21) encircles the "marked point" $\lambda=0$ together with the zeroes of $d z$. Using this formula, we derive in next section the perturbative prepotential. 
Thus, one can interpret the $5 d$ theory as the $4 d$ theory with infinite number of (Kaluza-Klein) vector multiplets with masses $M_{m}=\frac{m}{R_{5}}$ and infinite number of analogous (Kaluza-Klein) fundamental multiplets. Then, the presented $5 d$ picture naively corresponds to the infinitely covered " $4 d$ " (see the spectral curve equation in $\xi$-variable (3.14) living on a strip) also with infinitely many punctures. This, however, can be effectively encapsulated into hyperelliptic Riemann surface (3.11) with finite number of punctures, but, as a memory of infinitely many multiplets, the spectral parameter $\lambda$ now lives on a cylinder (on a sphere with two punctures). Meanwhile, the differential $d S^{(5)}$ should be now of the form (3.20) which "remembers" its $4 d$ origin.

Note that, in particular, the residue formula (3.21) can be written equally well in terms of variable $\xi$, instead of $\lambda$. Then, in the denominator of (3.21) there arises the expression $d z d \xi$ and the residue is taken only at zeroes of $d z$ (note also that this change of variables eliminates the factor 2 in (3.21)). This form of the residue formula looks considerably more symmetric and we use its analog for the $6 d$ case in sect.4.

\subsection{Perturbative limit of the SW anzatz}

In the perturbative limit, the hyperelliptic curve (3.11) turns into the rational curve

$$
W=2 \frac{P_{N_{c}}(\lambda)}{\sqrt{Q_{N_{c}}(\lambda)}} \equiv 2 \frac{\prod_{i}^{N_{c}}\left(\lambda-\lambda_{i}\right)}{\sqrt{\prod_{\alpha}^{N_{f}}\left(\lambda-\lambda_{\alpha}\right)}}
$$

with $\lambda_{i}=e^{2 a_{i}}, \sum_{i}^{N_{c}} a_{i}=0, \lambda_{\alpha}=e^{2 m_{\alpha}}$. We consider here the case of arbitrary number $N_{f} \geq 2 N_{c}$ of massive hypermultiplets. First, let us construct the set of holomorphic differentials $d \omega_{I}$.

The perturbative differential $d S$ is

$$
d S=\frac{1}{2} \log \lambda d \log \left(\frac{P(\lambda)}{\sqrt{Q(\lambda)}}\right)=\frac{1}{2} \log \lambda d \lambda\left(\sum_{i=1}^{N_{c}} \frac{1}{\lambda-\lambda_{i}}-\frac{1}{2} \sum_{\alpha=1}^{N_{f}} \frac{1}{\lambda-\lambda_{\alpha}}\right)
$$

or, equivalently,

$$
d S=\xi d \xi\left(\sum_{i=1}^{N_{c}} \operatorname{coth}\left(\xi-a_{i}\right)-\frac{1}{2} \sum_{\alpha=1}^{N_{f}} \operatorname{coth}\left(\xi-m_{\alpha}\right)+N_{c}-\frac{1}{2} N_{f}\right)
$$

The variation of (3.23) gives

$$
\begin{gathered}
2 \delta d S=-\log \lambda d\left(\sum_{i=1}^{N_{c}} \frac{\delta \lambda_{i}}{\lambda-\lambda_{i}}-\frac{1}{2} \sum_{\alpha=1}^{N_{f}} \frac{\delta \lambda_{\alpha}}{\lambda-\lambda_{\alpha}}\right) \cong \\
\cong \frac{d \lambda}{\lambda} \sum_{i=1}^{N_{c}-1} \frac{\delta \lambda_{i}}{\lambda_{i}}\left(\frac{\lambda_{i}}{\lambda-\lambda_{i}}-\frac{\lambda_{N_{c}}}{\lambda-\lambda_{N_{c}}}\right)-\frac{1}{2} \frac{d \lambda}{\lambda} \sum_{\alpha=1}^{N_{f}} \frac{\delta \lambda_{\alpha}}{\lambda_{\alpha}}\left(\frac{\lambda_{\alpha}}{\lambda-\lambda_{\alpha}}\right)
\end{gathered}
$$

where it is implied that only $N_{c}-1$ parameters $\lambda_{i}$ are independent so that $\frac{\delta \lambda_{N_{c}}}{\lambda_{N_{c}}}=-\sum_{i=1}^{N_{c}-1} \frac{\delta \lambda_{i}}{\lambda_{i}}$. Now the set of the $N_{c}-1$ independent canonical holomorphic differentials is

$$
d \omega_{i}=\frac{d \lambda}{\lambda}\left(\frac{\lambda_{i}}{\lambda-\lambda_{i}}-\frac{\lambda_{N_{c}}}{\lambda-\lambda_{N_{c}}}\right)=\frac{\lambda_{i N_{c}} d \lambda}{\left(\lambda-\lambda_{i}\right)\left(\lambda-\lambda_{N_{c}}\right)} \quad i=1, \ldots, N_{c}-1, \quad \lambda_{i j} \equiv \lambda_{i}-\lambda_{j}
$$

and one can check that the A-periods of $d S$ are $\log \lambda_{i}=2 a_{i}$, while the condition of vanishing the sum of all residues gives the relation (3.15) $\sum_{i} a_{i}=\frac{1}{2} \sum m_{\alpha}$. In s.3.1, we discussed how this strange 
constraint should be removed off by using the twisted $X X Z$ spin chain. The analytical explanation of this is that one can easily reproduce any lack of residues by putting some additional poles at infinities in $\xi$ or at $\lambda=0, \infty$. It is analogous to the procedure in $4 d$. We shall see, however, that the same procedure does not work in $d=6$.

The additional differentials corresponding to the derivatives of $d S$ (3.23) with respect to masses have the form 8

$$
d \omega_{\alpha}=-\frac{d \lambda}{2 \lambda} \frac{\lambda_{\alpha}}{\lambda-\lambda_{\alpha}}
$$

\subsection{Perturbative prepotentials}

Now, using the manifest expressions for the differentials, we can calculate the perturbative prepotentials using the residue formulas. Let us first consider the case of pure $S U\left(N_{c}\right) 5 d$ gauge theory, when the prepotential is defined by the following residue formulas [10]

$$
\mathcal{F}_{i j k}=2 \underset{d \log P=0}{\operatorname{res}} \frac{d \omega_{i} d \omega_{j} d \omega_{k}}{d \log P(\lambda) \frac{d \lambda}{\lambda}}
$$

since $\lambda=0$ does not contribute into the residues. These residues can be easily calculated if one deforms the contour around the poles of $d \omega_{i}$ 's. The final results have the following form

$$
\begin{gathered}
\mathcal{F}_{i i i}=\sum_{k \neq i} \operatorname{coth} a_{i k}+6 \operatorname{coth} a_{i N_{c}}+\sum_{k \neq N_{c}} \operatorname{coth} a_{k N_{c}}, \\
\mathcal{F}_{i i j}=-\operatorname{coth} a_{i j}+4 \operatorname{coth} a_{i N_{c}}+2 \operatorname{coth} a_{j N_{c}}+\sum_{k \neq i, j, N_{c}} \operatorname{coth} a_{k N_{c}}-N_{c}, \quad i \neq j, \\
\mathcal{F}_{i j k}=2 \sum_{l \neq N_{c}} \operatorname{coth} a_{l N_{c}}-\sum_{l \neq i, j, k, N_{c}} \operatorname{coth} a_{l N_{c}}-N_{c}, \quad i \neq j \neq k
\end{gathered}
$$

From these expressions it follows that the prepotential is given by formula

$$
\begin{gathered}
\mathcal{F}=\frac{1}{4} \sum_{i, j}\left(\frac{1}{3} a_{i j}^{3}-\frac{1}{2} \operatorname{Li}_{3}\left(e^{-2 a_{i j}}\right)\right)+\frac{N_{c}}{2} \sum_{i>j>k} a_{i} a_{j} a_{k}= \\
=\frac{1}{4} \sum_{i, j}\left(\frac{1}{3} a_{i j}^{3}-\frac{1}{2} \operatorname{Li}_{3}\left(e^{-2 a_{i j}}\right)\right)+\frac{N_{c}}{6} \sum_{i} a_{i}^{3}
\end{gathered}
$$

Now let us turn to the computation of the perturbative part of prepotential for the $5 d S U\left(N_{c}\right)$ theory with $N_{f}$ massive fundamental hypermultiplets and compare the results with the answers appeared in literature 23]. In order to include massive hypermultiplets, we use the residue formula

$$
\mathcal{F}_{I J K}=2 \underset{\substack{\operatorname{rog} \frac{P}{\sqrt{Q}} \\ \lambda=0}}{\operatorname{res}}=0 . \frac{d \omega_{I} d \omega_{J} d \omega_{K}}{d \log \frac{P(\lambda)}{\sqrt{Q(\lambda)}} \frac{d \lambda}{\lambda}}, \quad\{I, J, K, \ldots\}=\{i, j, k, \ldots \mid \alpha, \beta, \gamma, \ldots\}
$$

\footnotetext{
${ }^{8}$ It can be instructive to describe in detail why 2.29 arises not only in $4 d$, but also in $5 d$ models. Strictly speaking, in the $5 d$ case, one should consider differentials on annulus - on sphere with 2 marked points. Therefore, instead of $\frac{d \lambda}{\lambda-\lambda_{i}}$, one rather needs to take

$$
\sum_{m=-\infty}^{+\infty} \frac{d \xi}{\xi-a_{i}+m} \sim \operatorname{coth}\left(\xi-a_{i}\right) d \xi=\frac{\lambda+\lambda_{i}}{\lambda-\lambda_{i}} \frac{d \lambda}{2 \lambda}
$$

Taking now, instead of (2.29), the differentials (3.27)

$$
d \omega_{i}=\frac{\lambda+\lambda_{i}}{\lambda-\lambda_{i}} \frac{d \lambda}{2 \lambda}-\frac{\lambda+\lambda_{N_{c}}}{\lambda-\lambda_{N_{c}}} \frac{d \lambda}{2 \lambda}=\frac{\lambda_{i N_{c}} d \lambda}{\left(\lambda-\lambda_{i}\right)\left(\lambda-\lambda_{N_{c}}\right)}
$$

we obtain again the formula (2.29). On the other hand, the mass differentials (3.29) look different from 3.27). It turns out, however, that the difference does not contribute into the results and, therefore, one is free to choose any of these two possible mass differentials. We will return to this point in next subsection.
} 
and obtain

$$
\begin{gathered}
\frac{1}{2} \mathcal{F}_{i i i}=\sum_{k \neq i} \frac{\lambda_{i}}{\lambda_{i k}}+3 \frac{\lambda_{i}+\lambda_{N_{c}}}{\lambda_{i N_{c}}}+\sum_{k \neq N_{c}} \frac{\lambda_{N_{c}}}{\lambda_{k N_{c}}}+\frac{1}{2} \sum_{\alpha=1}^{N_{f}} \frac{\lambda_{i N_{c}} \lambda_{\alpha}}{\lambda_{i \alpha} \lambda_{N_{c} \alpha}} \\
\frac{1}{2} \mathcal{F}_{i i j}=1+2 \frac{\lambda_{N_{c}}}{\lambda_{i N_{c}}}+\frac{\lambda_{N_{c}}}{\lambda_{j N_{c}}}+\sum_{k \neq N_{c}} \frac{\lambda_{N_{c}}}{\lambda_{k N_{c}}}-\frac{\lambda_{i} \lambda_{j N_{c}}}{\lambda_{i N_{c}} \lambda_{i j}}+\frac{1}{2} \sum_{\alpha=1}^{N_{f}} \frac{\lambda_{N_{c}}}{\lambda_{N_{c} \alpha}}, \quad i \neq j \\
\frac{1}{2} \mathcal{F}_{i j k}=1+\sum_{l \neq N_{c}} \frac{\lambda_{N_{c}}}{\lambda_{l N_{c}}}+\sum_{l=i, j, k} \frac{\lambda_{N_{c}}}{\lambda_{l N_{c}}}+\frac{1}{2} \sum_{\alpha=1}^{N_{f}} \frac{\lambda_{N_{c}}}{\lambda_{N_{c} \alpha}}, \quad i \neq j \neq k \\
\mathcal{F}_{i i \alpha}=\frac{\lambda_{\alpha}}{\lambda_{i \alpha}}+\frac{\lambda_{\alpha}}{\lambda_{N_{c} \alpha}} \\
\mathcal{F}_{i j \alpha}=\frac{\lambda_{\alpha}}{\lambda_{N_{c} \alpha}} i \neq j \\
\mathcal{F}_{i \alpha \alpha}=\frac{\lambda_{i N_{c}} \lambda_{\alpha}}{\lambda_{i \alpha} \lambda_{N_{c} \alpha}} \\
\mathcal{F}_{\alpha \alpha \alpha}=1+\sum_{i=1}^{N_{c}} \frac{\lambda_{\alpha}}{\lambda_{i \alpha}}+\frac{1}{2} \sum_{\beta=1}^{N_{f}} \frac{\lambda_{\alpha}}{\lambda_{\alpha \beta}} \\
2 \mathcal{F}_{\alpha \alpha \beta}=-\frac{\lambda_{\beta}}{\lambda_{\alpha \beta}} \\
\mathcal{F}_{\alpha \beta \gamma}=\mathcal{F}_{i \alpha \beta}=0
\end{gathered}
$$

i.e.

$$
\begin{gathered}
\mathcal{F}_{i i i}=\sum_{k \neq i} \operatorname{coth} a_{i k}+6 \operatorname{coth} a_{i N_{c}}+\sum_{k \neq N_{c}} \operatorname{coth} a_{k N_{c}}+ \\
+\frac{1}{2} \sum_{\alpha}\left(\operatorname{coth}\left(a_{N_{c}}-m_{\alpha}\right)-\operatorname{coth}\left(a_{i}-m_{\alpha}\right)\right) \\
\mathcal{F}_{i i j}=-\operatorname{coth} a_{i j}+4 \operatorname{coth} a_{i N_{c}}+2 \operatorname{coth} a_{j N_{c}}+\sum_{k \neq i, j, N_{c}} \operatorname{coth} a_{k N_{c}}+\frac{1}{2} \sum_{\alpha} \operatorname{coth}\left(a_{N_{c}}-m_{\alpha}\right)- \\
-N_{c}+\frac{N_{f}}{2}, \quad i \neq j \\
\mathcal{F}_{i j k}=2 \sum_{l \neq N_{c}} \operatorname{coth} a_{l N_{c}}-\sum_{l \neq i, j, k, N_{c}} \operatorname{coth} a_{l N_{c}}+\frac{1}{2} \sum_{\alpha} \operatorname{coth}\left(a_{N_{c}}-m_{\alpha}\right)- \\
-N_{c}+\frac{N_{f}}{2}, \quad i \neq j \neq k \\
2 \mathcal{F}_{i i \alpha}=\operatorname{coth}\left(a_{i}-m_{\alpha}\right)+\operatorname{coth}\left(a_{N_{c}}-m_{\alpha}\right)-2 \\
2 \mathcal{F}_{i j \alpha}=\operatorname{coth}\left(a_{N_{c}}-m_{\alpha}\right)-1 \quad i \neq j \\
2 \mathcal{F}_{i \alpha \alpha}=\left(\operatorname{coth}\left(a_{N_{c}}-m_{\alpha}\right)-\operatorname{coth}\left(a_{i}-m_{\alpha}\right)\right) \\
2 \mathcal{F}_{\alpha \alpha \alpha}=\sum_{i} \operatorname{coth}\left(a_{i}-m_{\alpha}\right)+\frac{1}{2} \sum_{\beta \neq \alpha} \operatorname{coth}\left(m_{\alpha}-m_{\beta}\right)-N_{c}+\frac{N_{f}}{2}+\frac{3}{2} \\
4 \mathcal{F}_{\alpha \alpha \beta}=\operatorname{coth}\left(m_{\beta}-m_{\alpha}\right)-1 \\
\mathcal{F}_{i \alpha \beta}=\mathcal{F}_{\alpha \beta \gamma}=0
\end{gathered}
$$

The "strange" numbers $-1,-2, \frac{3}{2}$ that arise in the derivatives of the prepotential w.r.t. the mass moduli are due to the choice of the mass differentials in the form (3.29). Certainly, these numbers contribute 
into at most quadratic part of the prepotential in gauge moduli. This does not effect the "physical" implications but does break the WDVV equations [10]. Meanwhile, with the second possible choice (see footnote 5)

$$
d \omega_{\alpha}=-\frac{d \lambda}{4 \lambda} \frac{\lambda+\lambda_{\alpha}}{\lambda-\lambda_{\alpha}}=-\frac{d \xi}{2} \operatorname{coth}\left(\xi-m_{\alpha}\right)
$$

these strange number are no longer present in the derivatives - and this turns out to be the only influence of the replace of the differentials.

The difference between the two forms of mass differentials is due to different possible choices of the factor in the r.h.s. of (3.18). How it was already mentioned, this choice does not influence the result. It does, however, the WDVV equations.

Thus, the $5 d$ perturbative prepotential can be written in the following plausible form

$$
\begin{gathered}
\mathcal{F}=\frac{1}{4} \sum_{i, j} f^{(5)}\left(a_{i j}\right)-\frac{1}{4} \sum_{i, \alpha} f^{(5)}\left(a_{i}-m_{\alpha}\right)+\frac{1}{16} \sum_{\alpha, \beta} f^{(5)}\left(m_{\alpha}-m_{\beta}\right)+ \\
+\frac{1}{12}\left(2 N_{c}-N_{f}\right)\left(\sum_{i} a_{i}^{3}+\frac{1}{4} \sum_{\alpha} m_{\alpha}^{3}\right)
\end{gathered}
$$

where, as in (2.36) we use the functions $f^{(d)}(x)$ equal to

$$
f^{(5)}(x)=\frac{1}{3} x^{3}-\frac{1}{2} \operatorname{Li}_{3}\left(e^{-2 x}\right)
$$

\section{$4 \quad X Y Z$ chain and $6 d$ theories}

\subsection{General comments}

Now in the framework of general scheme developed in sect.2 and applied to the $5 d$ case in sect.3, we are going to turn to the $6 d$ case, or to the theory with two extra compactified dimensions, of radii $R_{5}$ and $R_{6}$. The same argument as we used for the transition from four to five dimensions, i.e. taking account of the Kaluza-Klein modes, allows one to predict the perturbative form of the charges in the $6 d$ case as well. Namely, one should expect them to have the form

$$
\begin{aligned}
& T_{i j} \sim \sum_{\text {masses }} \log \text { masses } \sim \sum_{m, n} \log \left(a_{i j}+\frac{m}{R_{5}}+\frac{n}{R_{6}}\right) \sim \\
& \sim \log \prod_{m, n}\left(R_{5} a_{i j}+m+n \frac{R_{5}}{R_{6}}\right) \sim \log \theta\left(R_{5} a_{i j} \mid i \frac{R_{5}}{R_{6}}\right)
\end{aligned}
$$

i.e. coming from $4 d(5 d)$ to $6 d$ one should replace the rational (trigonometric) expressions by the elliptic ones, at least, in the formulas for the perturbative prepotential, the (imaginary part of) modular parameter being identified with the ratio of the compactification radii $R_{5} / R_{6}$.

A similar change of variables would give rise to "elliptization" of an integrable systems. In terms of $2 \times 2$ Lax representation, the "elliptization" corresponds to replacing by elliptic $r$-matrices and $L$-operators the rational (2.1) and trigonometric (3.1) one. In particular, it means that now it is natural to consider parameters $\xi=\frac{1}{2} \log \lambda$ as a co-ordinate on torus, i.e. one of the bare spectral curves becomes a torus, while the other one remains to be a cylinder.

In fact, we know the only system providing proper elliptization of the $X X X$ spin chain, that is, the 
$X Y Z$ Sklyanin chain 18] 9. We propose it here as a candidate for the integrable system behind the $6 d$ theory. Below we describe the $X Y Z$ spin chain and demonstrate that it leads to the perturbative prepotential which coincides with our naive expectations (4.1), giving some support to our conjecture.

\section{2 $X Y Z$ chain}

Now we briefly describe the $X Y Z$ chain following [6]. All necessary facts on the elliptic functions can be found in Appendix. The Lax matrix is now defined on elliptic curve $E(\tau)$ and is explicitly given by (see [17] and references therein):

$$
L^{\mathrm{Skl}}(\xi)=S^{0} \mathbf{1}+i \frac{g}{\omega} \sum_{a=1}^{3} W_{a}(\xi) S^{a} \sigma_{a}
$$

where

$$
W_{a}(\xi)=\sqrt{e_{a}-\wp(\xi \mid \tau)}=i \frac{\theta_{*}^{\prime}(0) \theta_{a+1}(\xi)}{\theta_{a+1}(0) \theta_{*}(\xi)}
$$

To keep the similarities with [6], we redefine in this section the spectral parameter $\xi \rightarrow i \frac{\xi}{2 K}$, where $K \equiv \int_{0}^{\frac{\pi}{2}} \frac{d t}{\sqrt{1-k^{2} \sin ^{2} t}}=\frac{\pi}{2} \theta_{00}^{2}(0), k^{2} \equiv \frac{e_{1}-e_{2}}{e_{1}-e_{3}}$ so that $K \rightarrow \frac{\pi}{2}$ as $\tau \rightarrow i \infty$. This factor results into additional multiplier $\pi$ in the trigonometric functions in the limiting cases below.

The Lax operator (4.2) satisfies the Poisson relation (2.2) with the numerical elliptic r-matrix $r(\xi)=i \frac{g}{\omega} \sum_{a=1}^{3} W_{a}(\xi) \sigma_{a} \otimes \sigma_{a}$, which implies that $S^{0}, S^{a}$ form the (classical) Sklyanin algebra [18]:

$$
\begin{gathered}
\left\{S^{a}, S^{0}\right\}=2 i\left(\frac{g}{\omega}\right)^{2}\left(e_{b}-e_{c}\right) S^{b} S^{c} \\
\left\{S^{a}, S^{b}\right\}=2 i S^{0} S^{c}
\end{gathered}
$$

with the obvious notation: $a b c$ is the triple 123 or its cyclic permutations.

The coupling constant $\frac{g}{\omega}$ can be eliminated by simultaneous rescaling of the $S$-variables and the symplectic form:

$$
S^{a}=\frac{\omega}{g} \widehat{S}^{a} \quad S^{0}=\widehat{S}^{0} \quad\{,\} \rightarrow-2 \frac{g}{\omega}\{,\}
$$

Then

$$
\begin{gathered}
L^{\mathrm{Skl}}(\xi)=\widehat{S}^{0} \mathbf{1}+i \sum_{a=1}^{3} W_{a}(\xi) \widehat{S}^{a} \sigma_{a} \\
\left\{\widehat{S}^{a}, \widehat{S}^{0}\right\}=-i\left(e_{c}-e_{b}\right) \widehat{S}^{b} \widehat{S}^{c} \\
\left\{\widehat{S}^{a}, \widehat{S}^{b}\right\}=-i \widehat{S}^{0} \widehat{S}^{c}
\end{gathered}
$$

One can distinguish three interesting limits of the Sklyanin algebra: the rational, trigonometric and double-scaling limits [6]. We are interested in only trigonometric limit here, since it describes the degeneration to the $5 d$ case. In this limit, $\tau \rightarrow+i \infty$ and the Sklyanin algebra (4.7) transforms to

$$
\begin{gathered}
\left\{\widehat{S}^{3}, \widehat{S}^{0}\right\}=0, \quad\left\{\widehat{S}^{1}, \widehat{S}^{0}\right\}=i \widehat{S}^{2} \widehat{S}^{3}, \quad\left\{\widehat{S}^{2}, \widehat{S}^{0}\right\}=-2 i \widehat{S}^{3} \widehat{S}^{1} \\
\left\{\widehat{S}^{1}, \widehat{S}^{2}\right\}=-i \widehat{S}^{0} \widehat{S}^{3}, \quad\left\{\widehat{S}^{1}, \widehat{S}^{3}\right\}=i \widehat{S}^{0} \widehat{S}^{2}, \quad\left\{\widehat{S}^{2}, \widehat{S}^{3}\right\}=-i \widehat{S}^{0} \widehat{S}^{1}
\end{gathered}
$$

\footnotetext{
${ }^{9}$ In [6] it has been already proposed that the $X Y Z$ spin chain should have something to do with the $N_{f}=2 N_{c}$ SW theory. We will see below, that this is indeed the case: the condition $N_{f}=2 N_{c}$ which can be easily broken in $4 d$ and $5 d$ situations is very strict here. One may think of this theory as of blowing up all possible compactified dimensions in the $N_{f}=2 N_{c}$ theory with vanishing $\beta$-function, see the discussion below.
} 
The corresponding Lax matrix is

$$
L_{X X Z}=\widehat{S}^{0} \mathbf{1}-\frac{1}{\sinh \pi \xi}\left(\widehat{S}^{1} \sigma_{1}+\widehat{S}^{2} \sigma_{2}+\cosh \pi \xi \widehat{S}^{3} \sigma_{3}\right)
$$

and $r$-matrix

$$
r(\xi)=\frac{i}{\sinh \pi \xi}\left(\sigma_{1} \otimes \sigma_{1}+\sigma_{2} \otimes \sigma_{2}+\cosh \pi \xi \sigma_{3} \otimes \sigma_{3}\right)
$$

Now one can note that the algebra (4.8) admits the identification $\widehat{S}_{0}=e^{S_{0}^{\text {trig }}}+e^{-S_{0}^{\text {trig }}}, \widehat{S}_{3}=e^{-S_{0}^{\text {trig }}}-$ $e^{S_{0}^{\text {trig }}}$. With this identification and up to normalization of the Lax operator (4.9), we finally get the Lax operator (3.1) of the $X X Z$ chain with the Poisson brackets (3.4).

The determinant $\operatorname{det}_{2 \times 2} L^{\mathrm{Skl}}(\xi)$ is equal to

$$
\operatorname{det}_{2 \times 2} L^{\mathrm{Skl}}(\xi)=\widehat{S}_{0}^{2}+\sum_{a=1}^{3} e_{a} \widehat{S}_{a}^{2}-\wp(\xi) \sum_{a=1}^{3} \widehat{S}_{a}^{2}=K-M^{2} \wp(\xi)=K-M^{2} x
$$

where

$$
K=\widehat{S}_{0}^{2}+\sum_{a=1}^{3} e_{a}(\tau) \widehat{S}_{a}^{2} \quad M^{2}=\sum_{a=1}^{3} \widehat{S}_{a}^{2}
$$

are the Casimir operators of the Sklyanin algebra (i.e. Poisson commuting with all the generators $\widehat{S}^{0}$, $\left.\widehat{S}^{1}, \widehat{S}^{2}, \widehat{S}^{3}\right)$.

Now, in order to construct the spectral curve, note that the determinant of the monodromy matrix $T_{N_{c}}(\xi)=\prod_{N_{c} \geq i \geq 1}^{\curvearrowleft} L^{\mathrm{Skl}}\left(\xi-\xi_{i}\right)$ is

$$
\begin{gathered}
Q(\xi)=\operatorname{det}_{2 \times 2} T_{N_{c}}(\xi)=\prod_{i=1}^{N_{c}} \operatorname{det}_{2 \times 2} L^{\mathrm{Skl}}\left(\xi-\xi_{i}\right)=\prod_{i=1}^{N_{c}}\left(K_{i}-M_{i}^{2} \wp\left(\xi-\xi_{i}\right)\right)= \\
=Q_{0} \frac{\prod_{\alpha=1}^{N_{f}} \theta_{*}\left(\xi-m_{\alpha}\right)}{\prod_{i=1}^{N_{c}} \theta_{*}\left(\xi-\xi_{i}\right)^{2}}, \quad Q_{0}=\prod_{i}^{N_{c}} A_{i}
\end{gathered}
$$

where we used formulas (A.8), A.9) and (A.11) from Appendix in order to rewrite $K_{i}-M_{i}^{2} \wp\left(\xi-\xi_{i}\right)$ as $A_{i} \frac{\theta_{*}\left(\xi-m_{i}^{(+)}\right) \theta_{*}\left(\xi-m_{i}^{(-)}\right)}{\theta_{*}^{2}\left(\xi-\xi_{i}\right)}$. Here

$$
A_{i}=\frac{M_{i}^{2}\left[\theta_{*}^{\prime}(0)\right]^{2}}{\theta_{*}^{2}\left(\kappa_{i}\right)}, \quad m_{i}^{( \pm)}=\xi_{i} \pm \kappa_{i}
$$

and $\kappa_{i}$ is a solution to the equation

$$
\frac{\theta_{4}^{2}\left(\kappa_{i}\right)}{\theta_{*}^{2}\left(\kappa_{i}\right)}=\frac{K_{i} \theta_{4}^{2}(0)}{M_{i}^{2}\left[\theta_{*}^{\prime}(0)\right]^{2}}+\frac{\pi^{2} \theta_{4}^{2}(0)\left[\theta_{2}^{4}(0)+\theta_{3}^{4}(0)\right]}{3\left[\theta_{*}^{\prime}(0)\right]^{2}}
$$

Again

$$
\sum_{\alpha=1}^{N_{f}} m_{\alpha}=2 \sum_{i=1}^{N_{c}} \xi_{i}
$$

As a particular example, let us consider the case of the homogeneous chain [6] (all $\xi_{i}=0$ in (4.13) $\operatorname{Tr} T_{N_{c}}(\xi)$ is a combination of the polynomials:

$$
P(\xi)=\operatorname{Pol}_{\left[\frac{N_{c}}{2}\right]}^{(1)}(x)+y \operatorname{Pol}_{\left[\frac{N_{c}-3}{2}\right]}^{(2)}(x),
$$

where $\left[\frac{N_{c}}{2}\right]$ is integral part of $\frac{N_{c}}{2}$, and the coefficients of $P_{o l} l^{(1)}$ and $P o l^{(2)}$ are Hamiltonians of the $X Y Z$ model. The spectral equation (3.9) for the $X Y Z$ model is:

$$
w+\frac{Q(\xi)}{w}=2 P(\xi)
$$


where for the homogeneous chain $P$ and $Q$ are polynomials in $x=\wp(\xi)$ and $y=\frac{1}{2} \wp^{\prime}(\xi)$. Eq. (4.18) describes the double covering of the elliptic curve $E(\tau)$ : with generic point $\xi \in E(\tau)$ one associates the two points of $\Sigma^{X Y Z}$, labeled by two roots $w_{ \pm}$of equation (4.18). The ramification points correspond to $w_{+}=w_{-}= \pm \sqrt{Q}$, or $Y=\frac{1}{2}\left(w-\frac{Q}{w}\right)=\sqrt{P^{2}-Q}=0$. Note that, for the curve (4.18), $x=\infty$ is not a branch point, therefore, the number of cuts on the both copies of $E(\tau)$ is $N_{c}$ and the genus of the spectral curve is $N_{c}+1.10$

In the case of a generic inhomogeneous chain the trace of monodromy matrix looks more complicated, being expression of the form

$$
\begin{aligned}
\operatorname{Tr} T_{N_{c}}(\xi)=\operatorname{Tr} \prod_{N_{c} \geq i \geq 1}^{\curvearrowleft} L_{i}^{\mathrm{Skl}}\left(\xi-\xi_{i}\right)=\operatorname{Tr} \sum_{\left\{\alpha_{i}\right\}} \prod_{N_{c} \geq i \geq 1}^{\curvearrowleft} \sigma^{\alpha_{i}} S_{i}^{\alpha_{i}} W_{\alpha_{i}}\left(\xi-\xi_{i}\right)= \\
\quad=\sum_{\left\{\alpha_{i}\right\}} \operatorname{Tr} \prod_{N_{c} \geq i \geq 1}^{\curvearrowleft} \sigma^{\alpha_{i}} S_{i}^{\alpha_{i}} W_{\alpha_{i}}\left(\xi-\xi_{i}\right) \\
\alpha_{i}=0, a_{i} ; \quad a_{i}=1,2,3 ; \quad W_{0}(\xi)=1
\end{aligned}
$$

It is clear from (4.3) that each term in the sum (4.21) (and thus the whole sum) has simple poles in all inhomogeneities $\xi=\xi_{i}$. Thus the trace of the monodromy matrix can be represented as

$$
\begin{gathered}
P(\xi)=\frac{1}{2} \operatorname{Tr} T_{N_{c}}(\xi)=P_{0} \frac{\prod_{i=1}^{N_{c}} \theta_{*}\left(\xi-a_{i}\right)}{\prod_{i=1}^{N_{c}} \theta_{*}\left(\xi-\xi_{i}\right)} \\
\sum_{i} a_{i}=\sum_{i} \xi_{i}=\frac{1}{2} \sum_{\alpha} m_{\alpha}
\end{gathered}
$$

where $a_{i}$ should be identified with (shifted) gauge moduli - see discussion in sect.3.1. The equation (4.18) can be finally rewritten as

$$
w+Q_{0} \frac{\prod_{\alpha=1}^{N_{f}} \theta_{*}\left(\xi-m_{\alpha}\right)}{w \prod_{i=1}^{N_{c}} \theta_{*}\left(\xi-\xi_{i}\right)^{2}}=P_{0} \frac{\prod_{i=1}^{N_{c}} \theta_{*}\left(\xi-a_{i}\right)}{\prod_{i=1}^{N_{c}} \theta_{*}\left(\xi-\xi_{i}\right)}
$$

We see that in $6 d$ case it is necessary always to require $N_{f}=2 N_{c}$ in order to have a single-valued meromorphic functions on torus. Again we also meet the constraint (4.23). In the $5 d$ case, it was removed, along with the condition $N_{f}=2 N_{c}$ twisting the spin chain. Since no twisted $X Y Z$ chain is known we are forced to preserve both constraints, their origin will be discussed below 11 .

The curve (4.24) has no longer that simple expression in terms of polynomials of $x$ and $y$ similar to (4.17). Thus, in the inhomogeneous case it is not convenient to work in terms of "elliptic" variables $x$ and $y$ (analogous to $\lambda$ in the $5 d$ case) instead we choose to deal with the variable $\xi \in \mathbf{C} / \Gamma$.

${ }^{10}$ Rewriting analytically $\Sigma^{X Y Z}$ as a system of equations

$$
y^{2}=\prod_{a=1}^{3}\left(x-e_{a}\right), \quad Y^{2}=P^{2}-Q
$$

the set of holomorphic 1-differentials on $\Sigma^{X Y Z}$ can be chosen as

$$
v=\frac{d x}{y}, \quad V_{\alpha}=\frac{x^{\alpha} d x}{y Y} \quad \alpha=0, \ldots,\left[\frac{N_{c}}{2}\right], \quad \widetilde{V}_{\beta}=\frac{x^{\beta} d x}{Y} \quad \beta=0, \ldots,\left[\frac{N_{c}-3}{2}\right]
$$

with the total number of holomorphic 1-differentials $1+\left(\left[\frac{N_{c}}{2}\right]+1\right)+\left(\left[\frac{N_{c}-3}{2}\right]+1\right)=N_{c}+1$ being equal to the genus of $\Sigma^{X Y Z}$

${ }^{11}$ Formula (4.23) implies that, while the sum of all the would-be gauge moduli $a_{i}$ still remains a constant, it is no longer zero. Therefore, one would rather associate with the gauge moduli the quantities $a_{i}$ shifted by the constant $\frac{1}{2 N_{c}} \sum m_{\alpha}$. We do not know the proper field theory interpretation of this condition. 
Given the spectral curve and integrable system one can immediately write down the generating 1-differential $d S$. It can be chosen in the following way

$$
d S^{X Y Z} \cong \xi \frac{d w}{w} \cong-\log w d \xi
$$

Return to our oversimplified example of the homogeneous chain. Now, under the variation of moduli (which are all contained in $P$, while $Q$ is moduli independent),

$$
\delta\left(d S^{X Y Z}\right) \cong \frac{\delta w}{w} d \xi=\frac{\delta P(\xi)}{\sqrt{P(\xi)^{2}-4 Q(\xi)}} d \xi=\frac{d x}{y Y} \delta P
$$

and, according to (4.20), the r.h.s. is a holomorphic 1-differential on the spectral curve (4.18).

The residue formula in the $6 d$ case is of the form completely analogous to the $4 d$ case (and to the $5 d$ case, when working in terms of the variable $\xi$ )

$$
\frac{\partial^{3} \mathcal{F}}{\partial a_{I} \partial a_{J} \partial a_{K}}=\operatorname{res}_{d z=0} \frac{d \omega_{I} d \omega_{J} d \omega_{K}}{d z d \xi}
$$

and we use it in the next subsections to derive the perturbative prepotential.

\subsection{Perturbative limit of the XYZ spin chain}

A generic $X Y Z$ curve (4.24) in the perturbative limit turns into

$$
W=h_{0} \frac{\prod_{i=1}^{N_{c}} \theta_{*}\left(\xi-a_{i}\right)}{\sqrt{\prod_{\alpha=1}^{N_{f}} \theta_{*}\left(\xi-m_{\alpha}\right)}}
$$

where $h_{0}=\frac{2 P_{0}}{\sqrt{Q_{0}}}$. Then, the rescaled generating differential 4.25 is

$$
\begin{array}{r}
d S=\left(\sum \log \theta_{*}\left(\xi-a_{i}\right)-\frac{1}{2} \sum \log \theta_{*}\left(\xi-m_{a}\right)\right) d \xi+\log h_{0} d \xi \cong \\
\cong \xi d \xi\left(\sum_{i=1}^{N_{c}} \zeta\left(\xi-a_{i}\right)-\frac{1}{2} \sum_{\alpha=1}^{N_{f}} \zeta\left(\xi-m_{\alpha}\right)\right)
\end{array}
$$

where the linear piece disappears due to $\sum a_{i}=\frac{1}{2} \sum m_{\alpha}$. This expression should be compared with (3.24) in the $5 d$ case.

Note that the sum of residues vanishes only provided the constraint 4.23 is fulfilled. It can not be removed by adding an additional pole at infinity, since the variable $\xi$, in contrast to the $4 d$ and $5 d$ cases, lives on the compact surface with out boundaries (torus) and, therefore, is always finite.

Now one can take the total variation of $d S$ (we neglect the trivial variation $\delta h_{0}$ giving rise to the holomorphic differential $d \xi$ on the bare torus)

$$
\delta d S^{X Y Z} \cong \sum_{i=1}^{N_{c}} \bar{\zeta}\left(\xi-a_{i}\right) \delta a_{i} d \xi-\frac{1}{2} \sum_{\alpha=1}^{N_{f}} \bar{\zeta}\left(\xi-m_{\alpha}\right) \delta m_{\alpha} d \xi
$$

where $\bar{\zeta}(\xi \mid \tau)$ is defined in Appendix (see (A.6)). Therefore, the differentials related to gauge moduli are

$$
d \omega_{i}=\left(\bar{\zeta}\left(\xi-a_{i}\right)-\bar{\zeta}\left(\xi-a_{N_{c}}\right)\right) d \xi
$$


while those related to masses are

$$
d \omega_{\alpha}=-\frac{1}{2}\left(\bar{\zeta}\left(\xi-m_{\alpha}\right)-\bar{\zeta}\left(\xi-a_{N_{c}}\right)\right) d \xi
$$

The second term in this expression is due to the condition (4.23). Thus, one can see that, in the $6 d$ case, mass moduli are practically identical to the gauge ones.

The residue formula acquires the form

$$
\mathcal{F}_{I J K}=\operatorname{res}_{d \log \frac{P}{\sqrt{Q}}=0} \frac{d \omega_{I} d \omega_{J} d \omega_{K}}{d \xi d \log \frac{P}{\sqrt{Q}}}
$$

\subsection{Perturbative prepotentials}

Having the explicit formulas for the differentials (4.31) and (4.32), one can use the residue formula (4.33) to calculate the perturbative prepotential in the $6 d$ case. The result is

$$
\begin{aligned}
& \mathcal{F}_{i i i}=\sum_{k \neq i} \bar{\zeta}\left(a_{i k}\right)+6 \bar{\zeta}\left(a_{i N_{c}}\right)+\sum_{k \neq N_{c}} \bar{\zeta}\left(a_{k N_{c}}\right)+ \\
& +\frac{1}{2} \sum_{\alpha}\left(\bar{\zeta}\left(a_{N_{c}}-m_{\alpha}\right)-\bar{\zeta}\left(a_{i}-m_{\alpha}\right)\right) \\
& \mathcal{F}_{i i j}=-\bar{\zeta}\left(a_{i j}\right)+4 \bar{\zeta}\left(a_{i N_{c}}\right)+2 \bar{\zeta}\left(a_{j N_{c}}\right)+\sum_{k \neq i, j, N_{c}} \bar{\zeta}\left(a_{k N_{c}}\right)+\frac{1}{2} \sum_{\alpha} \bar{\zeta}\left(a_{N_{c}}-m_{\alpha}\right), \quad i \neq j \\
& \mathcal{F}_{i j k}=2 \sum_{l \neq N_{c}} \bar{\zeta}\left(a_{l N_{c}}\right)-\sum_{l \neq i, j, k, N_{c}} \bar{\zeta}\left(a_{l N_{c}}\right)+\frac{1}{2} \sum_{\alpha} \bar{\zeta}\left(a_{N_{c}}-m_{\alpha}\right), \quad i \neq j \neq k \\
& 2 \mathcal{F}_{i i \alpha}=\bar{\zeta}\left(a_{i}-m_{\alpha}\right)+\bar{\zeta}\left(a_{N_{c}}-m_{\alpha}\right)+\bar{\zeta}\left(a_{N_{c} i}\right)+\sum_{k}^{N_{c}} \bar{\zeta}\left(a_{N_{c} k}\right)-\frac{1}{2} \sum_{\beta}^{N_{f}} \bar{\zeta}\left(a_{N_{c}}-m_{\beta}\right) \\
& 2 \mathcal{F}_{i j \alpha}=\bar{\zeta}\left(a_{N_{c}}-m_{\alpha}\right)+\bar{\zeta}\left(a_{N_{c} i}\right)+\bar{\zeta}\left(a_{N_{c} j}\right)+\sum_{k}^{N_{c}} \bar{\zeta}\left(a_{N_{c} k}\right)-\frac{1}{2} \sum_{\beta}^{N_{f}} \bar{\zeta}\left(a_{N_{c}}-m_{\beta}\right) \quad i \neq j \\
& 2 \mathcal{F}_{i \alpha \alpha}=\bar{\zeta}\left(a_{i}-m_{\alpha}\right)+\frac{1}{2} \bar{\zeta}\left(a_{i N_{c}}\right)-\frac{1}{2}\left(\sum_{k}^{N_{c}} \bar{\zeta}\left(a_{N_{c} k}\right)-\frac{1}{2} \sum_{\beta}^{N_{f}} \bar{\zeta}\left(a_{N_{c}}-m_{\beta}\right)\right) \\
& 2 \mathcal{F}_{\alpha \alpha \alpha}=\sum_{i} \bar{\zeta}\left(a_{i}-m_{\alpha}\right)+\frac{1}{2} \sum_{\beta \neq \alpha} \bar{\zeta}\left(m_{\alpha}-m_{\beta}\right)+\frac{1}{4}\left(\sum_{k}^{N_{c}} \bar{\zeta}\left(a_{N_{c} k}\right)-\frac{1}{2} \sum_{\beta}^{N_{f}} \bar{\zeta}\left(a_{N_{c}}-m_{\beta}\right)\right)+ \\
& +\frac{3}{4} \bar{\zeta}\left(m_{\alpha}-a_{N_{c}}\right) \\
& 4 \mathcal{F}_{\alpha \alpha \beta}=\bar{\zeta}\left(m_{\beta}-m_{\alpha}\right)+\frac{1}{2} \bar{\zeta}\left(m_{\alpha}-a_{N_{c}}\right)+\frac{1}{2}\left(\sum_{k}^{N_{c}} \bar{\zeta}\left(a_{N_{c} k}\right)-\frac{1}{2} \sum_{\beta}^{N_{f}} \bar{\zeta}\left(a_{N_{c}}-m_{\beta}\right)\right) \\
& 4 \mathcal{F}_{i \alpha \beta}=\bar{\zeta}\left(m_{\alpha}-a_{N_{c}}\right)+\bar{\zeta}\left(m_{\beta}-a_{N_{c}}\right)+\bar{\zeta}\left(a_{i N_{c}}\right)-\sum_{k}^{N_{c}} \bar{\zeta}\left(a_{N_{c} k}\right)-\frac{1}{2} \sum_{\beta}^{N_{f}} \bar{\zeta}\left(a_{N_{c}}-m_{\beta}\right) \\
& 8 \mathcal{F}_{\alpha \beta \gamma}=-\bar{\zeta}\left(m_{\alpha}-a_{N_{c}}\right)-\bar{\zeta}\left(m_{\beta}-a_{N_{c}}\right)-\bar{\zeta}\left(a_{i N_{c}}\right)+\sum_{k}^{N_{c}} \bar{\zeta}\left(a_{N_{c} k}\right)-\frac{1}{2} \sum_{\beta}^{N_{f}} \bar{\zeta}\left(a_{N_{c}}-m_{\beta}\right)
\end{aligned}
$$


These formulas imply that the perturbative prepotential is given by

$$
\mathcal{F}=\frac{1}{4} \sum_{i, j} f^{(6)}\left(a_{i j}\right)-\frac{1}{4} \sum_{i, \alpha} f^{(6)}\left(a_{i}-m_{\alpha}\right)+\frac{1}{16} \sum_{\alpha, \beta} f^{(6)}\left(m_{\alpha}-m_{\beta}\right)
$$

where $\sum a_{i}=\frac{1}{2} \sum m_{\alpha}$ and the function $f^{(6)}(x)$ defined so that

$$
f^{(6)^{\prime \prime}}(x)=\log \theta_{*}(x)
$$

can be expressed through the elliptic tri-logarithm $\mathrm{Li}_{3, q}(x)$ [24]:

$$
\begin{gathered}
f^{(6)}(a)=\sum_{m, n} f^{(4)}\left(a+\frac{n}{R_{5}}+\frac{m}{R_{6}}\right)=\sum_{n} f^{(5)}\left(a+n \frac{R_{5}}{R_{6}}\right)= \\
=\left(\frac{1}{3} a^{3}-\frac{a^{2}}{4}+\frac{1}{120}-\frac{1}{2} \operatorname{Li}_{3, q}\left(e^{-2 a}\right)\right)
\end{gathered}
$$

\section{Discussion}

Now we are going to discuss the results. We have reproduced the spectral curves for the SUSY $S U\left(N_{c}\right)$ gauge theories with $N_{f}$ massive hypermultiplets in $d=4,5$ and 6 dimensions. Using these curves and the corresponding differentials, we have calculated the perturbative parts of the prepotentials in all these cases and the results have the same form.

Results. The spectral curve in all cases can be written in the form

$$
w+\frac{Q^{(d)}(\xi)}{w}=2 P^{(d)}(\xi)
$$

or

$$
W+\frac{1}{W}=\frac{2 P^{(d)}(\xi)}{\sqrt{Q^{(d)}(\xi)}}, \quad W=\frac{w}{\sqrt{Q^{(d)}(\xi)}}
$$

The generating differential $d S$ is always of the form

$$
d S=\xi d \log W
$$

The perturbative part of the prepotential is always of the form

$$
\mathcal{F}=\frac{1}{4} \sum_{i, j} f^{(d)}\left(a_{i j}\right)-\frac{1}{4} \sum_{i, \alpha} f^{(d)}\left(a_{i}-m_{\alpha}\right)+\frac{1}{16} \sum_{\alpha, \beta} f^{(d)}\left(m_{\alpha}-m_{\beta}\right)
$$

The concrete forms of the functions introduced here are:

$$
\begin{aligned}
& Q^{(4)}(\xi) \sim \prod_{\alpha}^{N_{f}}\left(\xi-m_{\alpha}\right), \quad Q^{(5)}(\xi) \sim \prod_{\alpha}^{N_{f}} \sinh \left(\xi-m_{\alpha}\right), \quad Q^{(6)}(\xi) \sim \prod_{\alpha}^{N_{f}} \frac{\theta_{*}\left(\xi-m_{\alpha}\right)}{\theta_{*}^{2}\left(\xi-\xi_{i}\right)} \\
& P^{(4)} \sim \prod_{i}^{N_{c}}\left(\xi-a_{i}\right), \quad P^{(5)} \sim \prod_{i}^{N_{c}} \sinh \left(\xi-a_{i}\right), \quad P^{(6)} \sim \prod_{i}^{N_{c}} \frac{\theta_{*}\left(\xi-a_{i}\right)}{\theta_{*}\left(\xi-\xi_{i}\right)}
\end{aligned}
$$

(in $P^{(5)}(\xi)$, there is also some exponential of $\xi$ unless $N_{f}=2 N_{c}$, see (3.18)

$$
\begin{gathered}
f^{(4)}(x)=x^{2} \log x, \quad f^{(5)}(x)=\sum_{n} f^{(4)}\left(x+\frac{n}{R_{5}}\right)=\frac{1}{3}\left|x^{3}\right|-\frac{1}{2} \operatorname{Li}_{3}\left(e^{-2|x|}\right), \\
f^{(6)}(x)=\sum_{m, n} f^{(4)}\left(x+\frac{n}{R_{5}}+\frac{m}{R_{6}}\right)=\sum_{n} f^{(5)}\left(x+n \frac{R_{5}}{R_{6}}\right)= \\
=\left(\frac{1}{3}\left|x^{3}\right|-\frac{1}{2} \operatorname{Li}_{3, q}\left(e^{-2|x|}\right)+\text { quadratic terms }\right)
\end{gathered}
$$


so that

$$
f^{(4)^{\prime \prime}}=\log x, \quad f^{(5)^{\prime \prime}}(x)=\log \sinh x, \quad f^{(6)^{\prime \prime}}(x)=\log \theta_{*}(x)
$$

Note that, in the $6 d$ case, $N_{f}$ is always equal to $2 N_{c}$. The variables $\xi_{i}$ above are inhomogeneities in integrable system, and, in $d=5,6$, there is a restriction $\sum a_{i}=\sum \xi_{i}=\frac{1}{2} \sum m_{\alpha}$ which implies that the gauge moduli would be rather associated with $a_{i}$ shifted by the constant $\frac{1}{2 N_{c}} \sum m_{\alpha}$.

On perturbative limit. Now let us make some comments concerning the perturbative limit in all these cases. In fact, the procedure is quite clear in the $4 d$ and $5 d$ cases: this limit corresponds to big values of gauge moduli (and masses) compare to $\Lambda_{Q C D}$ and can be taken as $\frac{\Lambda_{Q C D}}{\mu} \rightarrow 0$, which is exactly the perturbative limit in asymptotically free theories. Looking at (5.2), one can see that this procedure corresponds to $\xi$ going to infinity (as $\Lambda_{Q C D}^{-1}$ ) and $W$ going to infinity or to zero depending on the choice of a particular list of hyperelliptic spectral curve 12. From the point of view of the effective charges $T_{i j}$ it separates logarithmic terms from the power (instantonic) contributions.

This reasoning certainly fails when $d=6$. Indeed, since $\xi \in \mathbf{C} / \Gamma$ it can not be taken to infinity. In terms of prepotential it means that one can not distinguish between $\log \theta$ terms and the other since $\theta$-function is restricted. However, there is a way out of this problem, although a little bit artificial. Namely, instead of moving $\xi$ one may just turn to zero the coefficient in front of the product in $Q^{(6)}(\xi)$. The perturbative contribution to the $6 d$ prepotential calculated in the paper correspond exactly to this limit.

As it is well-known, $5 d$ and $6 d$ theories are non-renormalizable. Therefore, the status of the perturbative contributions has to be understood better (see also [23, 25, 8, 26]). We are not going to discuss this question in detail here, instead let us point out that the problems of renormalizability are related only with maximally quadratic part of the prepotential getting stronger than logarithmic divergencies in $5 d$ and $6 d$ perturbative theory. Our approach does not control these terms and the properties of (compactified) $5 d$ and $6 d$ theories we discuss do not depend on quadratic contributions.

The first property is the periodicity of the $5 d$ prepotential under $a_{i} \rightarrow a_{i}+2 i \pi n R_{5}{ }^{-1}$, with integer $n$, which is evidently correct up to quadratic terms (being changed due to the shifts in cubic terms). This shift is induced by changing the sheet of the $\log$ arithm in $d S^{(5)}$ (3.20) (either $\log \lambda$, or $\log w$, depending on the chosen form of $\left.d S^{(5)}\right)$ as one can easily obtain from (2.25). It explains, in particular, why the ambiguity in definition of $d S^{(5)}(3.20)$ does not influence the result. Similarly, in $6 d$ case one needs double-periodicity to guarantee the unambiguous prepotential despite the multi-valuedness of generating differential $d S^{(6)}(4.25)$. This is indeed the case: $f^{(6)}$ in (4.37) is a double-periodic function.

The second property of the prepotentials is typically to satisfy the WDVV equations [9, 10]. This is strongly related to integrable structure of the theory and should have a topological origin. The WDVV equations are equations for the third derivatives and do not depend on quadratic terms. On the other hand, these equations requires to consider masses and gauge moduli on equal footing. Therefore, the WDVV equations are sensible to the constant corrections to the third derivatives of the prepotential, even if some of these derivatives are taken w.r.t. masses.

This issue has a lot of to do with the proper definition of mass differentials (see sect.3), we are

\footnotetext{
${ }^{12}$ In $4 d W \rightarrow \infty$ as $\Lambda_{Q C D}^{\frac{1}{2} N_{f}-N_{c}}$. However, one can renormalize the gauge moduli, masses, $W$ and $\xi$ so that they remain finite as $\Lambda_{Q C D} \rightarrow 0$. It results into the coefficient $\Lambda_{Q C D}^{2 N_{c}-N_{f}}$ in front of the term $\frac{1}{W}$ in 5.2 . The analogous procedure can be also performed for the other sheet, when $W \rightarrow 0$. In $5 d$ the dependence on $\Lambda_{Q C D}$ can not be reduced to the only coefficient, this is an indication of non-renormalizability of $5 d$ theory.
} 
going to return to this problem elsewhere.

$5 d$ and $6 d$ theories - cubic terms, $N_{f}=2 N_{c}$ etc. Next point we are going to discuss is the cubic (in moduli) terms appearing only in higher-dimensional theories. First, take the $5 d$ case, where these terms corresponds to the Chern-Simons term in the field theory Lagrangian $\operatorname{Tr}(A \wedge F \wedge F)$. In our computation they appear due to the constant piece $\left(N_{c}-\frac{1}{2} N_{f}\right)$ in the brackets in (3.24).

In fact, let us choose the special values of the second Casimir functions at $m$ sites so that the polynomial $Q(\lambda)$ acquires the form $\lambda^{m} \bar{Q}_{N_{f}}(\lambda)$, i.e. $m$ of $2 N_{c}$ factors in (3.12) turn into $\lambda^{m}$. In this case one gets the constant piece $N_{c}-m-N_{f} / 2$ in $d S^{(5)}$, with an integer $m \leq 2 N_{c}-N_{f}$. Therefore, the coefficient in front of cubic term in (3.37) can be made equal to $\frac{1}{6}\left(N_{c}-m-N_{f} / 2\right)$.

Restoring the dependence on $R_{5}$ in (3.37) $\left(a_{i} \rightarrow a_{i} R_{5}\right.$ and $\left.m_{\alpha} \rightarrow m_{\alpha} R_{5}\right)$ one can study the different limits of the system. The simplest limit corresponds to $4 d$ case and is given by $R_{5} \rightarrow 0$. In this limit, $f^{(5)}(x) \underset{x \sim 0}{\rightarrow} f^{(4)}(x)$, the cubic terms vanish and we reproduce the perturbative $4 d$ prepotential (2.36). At the level of integrable system it is enough to replace $S_{i} \rightarrow R_{5} S_{i}, \mu \rightarrow e^{R_{5} \mu}$ in the Lax operator (3.1) $(3.16)$ ) in order to reproduce the Lax operator of the $X X X$ (higher $s l(p)$ ) spin chain, see [15] for details).

Another interesting limit is the limit of flat $5 d$ space-time, i.e. $R_{5} \rightarrow \infty$. In this limit, only cubic terms survive in the prepotential (3.37) (one should carefully fix the branch of $f^{(6)}(x)$ which leading to appearing of the absolute value in $(5.9))$ :

$$
\begin{gathered}
\mathcal{F}=\frac{1}{12} \sum_{i, j}\left|a_{i j}\right|^{3}-\frac{1}{12} \sum_{i, \alpha}\left|a_{i}+m_{\alpha}\right|^{3}+\frac{1}{48} \sum_{\alpha, \beta}\left|m_{\alpha}-m_{\beta}\right|+ \\
+\frac{1}{12}\left(2 N_{c}-N_{f}\right)\left(\sum_{i} a_{i}^{3}+\frac{1}{4} \sum_{\alpha} m_{\alpha}^{3}\right)
\end{gathered}
$$

In fact, there are two different sources of cubic terms [23]. The first one is from the function $f^{(5)}(x)$. Since this function can be obtained as the sum of the $4 d$ perturbative contributions over the KaluzaKlein modes, these cubic terms have perturbative origin and come from the 1-loop (due to the wellknown effect of generation of the CS terms in odd-dimensional gauge theories).

The second source of the cubic terms is due to the bare CS Lagrangian. As it was shown in [23], one can consider these terms with some coefficient $c_{c l}$ :

$$
\frac{c_{c l}}{6} \sum_{i} a_{i}^{3}
$$

restricted only to satisfy the quantization condition $c_{c l}+\frac{N_{f}}{2} \in \mathbb{Z}$ and the inequality $\left|c_{c l}\right| \geq N_{c}-\frac{N_{f}}{2}$. In our formulas, we easily reproduce these conditions so that (5.9) coincides with [23], provided the prepotential is defined in a fixed Weyl chamber. We leave, however, the wall crossing jumps out of the discussion. Note that the curve (3.11) with this arbitrary $m$ coincides with that proposed in [22].

Note also that it is clear why the WDVV equations are not necessarily satisfied when $m \neq 0$. Since it corresponds to degeneration of an integrable system, one fixes some moduli (masses) - the parameters to vary in the WDVV equations (see [10] for details).

In the $6 d$ case the calculations are quite similar, and one would expect the similar cubic terms correspond to $\operatorname{Tr}(F \wedge F \wedge F)$. However, despite all the difference in calculations is due to slightly different mass differentials (3.36) and (4.32) the bare cubic terms in $6 d$ are absent. This occurs due to $N_{f}=2 N_{c}$ (since the coefficient in front of cubic terms is proportional to $N_{c}-\frac{1}{2} N_{f}$ ) and, therefore, 
cancelation of the constant term in the brackets of (4.29) as compared with (3.24). This is one of the problems with $6 d$ theories: one can not deform (twist) the theory so that the constraints $N_{f}=2 N_{c}$ and (4.23) look unavoidable. We do not understand clearly their meaning at the level of field theory. As for the "quantum generated" terms, i.e. those coming from the function $f^{(6)}$, they are certainly presented, with the coefficient equal to that in the $5 d$ case.

Finally, let us point out that the Lax matrices (2.1), (3.1) and (4.2) (and analogously though more involved for the transfer matrix (4.21) $)$ can be possibly determined from a linear differential equations $\bar{D} L_{i}(\xi) \sim \delta^{(2)}\left(\xi-\xi_{i}\right)$ (a lá Hitchin). These equations could be interpreted as arising in the framework of D-brane interpretation of SUSY gauge theories [12, 14]. It might indicate that the standard Hitchin-like systems and the spin-chain like models, in spite of distinctions in the formulation of the Poisson structures etc [17], can be treated within some unified approach in the framework of higher-dimensional SUSY gauge theories and corresponding brane configurations of string theory. We are going to return to this problem elsewhere.

\section{Appendix}

The definition of elliptic functions we use throughout the paper slightly differs from standard definitions that can be found, for example, in [27]. In this Appendix we define all the functions we need and write down manifestly some useful identities necessary for sect.4.

We define the Weierstrass $\wp$-function to be the sum

$$
\wp(\xi \mid \tau)=\sum_{m, n=-\infty}^{+\infty} \frac{1}{(\xi+m+n \tau)^{2}}-\sum_{m, n=-\infty}^{+\infty} \frac{1}{(m+n \tau)^{2}}=\frac{1}{\xi^{2}}+\mathcal{O}\left(\xi^{2}\right)
$$

where $\sum^{\prime}$ means omitted term with $m=n=0$. Thus defined $\wp$-function is double periodic, with periods 1 and $\tau=\frac{\omega}{\omega^{\prime}}$ and differs from the standard definition by the factor $(2 \omega)^{-2}$ and rescaling $\xi \rightarrow 2 \omega \xi$. According to this definition, the values of $\wp(\xi \mid \tau)$ in the half-periods, $e_{a}=e_{a}(\tau), a=1,2,3$, also differ by the factor $(2 \omega)^{-2}$ from the standard definition.

The complex torus $E(\tau)$ can be defined as $\mathbb{C} / \mathbb{Z} \oplus \tau \mathbb{Z}$ with a flat co-ordinate $\xi$ defined modulo $(1, \tau)$. Alternatively, any torus (with a marked point) can be described as the elliptic curve

$$
y^{2}=\left(x-e_{1}\right)\left(x-e_{2}\right)\left(x-e_{3}\right), \quad x=\wp(\xi), \quad y=\frac{1}{2} \wp^{\prime}(\xi)
$$

and the canonical holomorphic 1-differential is

$$
d \xi=2 \frac{d x}{y}
$$

Other elliptic functions used in the paper are $\zeta$-function

$$
\zeta(\xi \mid \tau)=\frac{1}{\xi}+\sum_{m, n=-\infty}^{+\infty}\left(\frac{1}{\xi-m-n \tau}+\frac{1}{m+n \tau}+\frac{\xi}{(m+n \tau)^{2}}\right)=\frac{1}{\xi}+\mathcal{O}\left(\xi^{3}\right)
$$


and $\theta$-functions

$$
\begin{gathered}
\theta_{1}(\xi \mid \tau)=\theta_{11}(\xi \mid \tau)=\theta_{*}(\xi \mid \tau)=i \sum_{n=-\infty}^{+\infty}(-1)^{n} e^{i\left(n-\frac{1}{2}\right)^{2} \pi \tau} e^{i \pi(2 n-1) \xi} \\
\theta_{2}(\xi \mid \tau)=\theta_{01}(\xi \mid \tau)=\sum_{n=-\infty}^{+\infty} e^{i\left(n-\frac{1}{2}\right)^{2} \pi \tau} e^{i \pi(2 n-1) \xi} \\
\theta_{3}(\xi \mid \tau)=\theta_{00}(\xi \mid \tau)=\sum_{n=-\infty}^{+\infty} e^{i n^{2} \pi \tau} e^{i \pi 2 n \xi} \\
\theta_{4}(\xi \mid \tau)=\theta_{10}(\xi \mid \tau)=\sum_{n=-\infty}^{+\infty}(-1)^{n} e^{i n^{2} \pi \tau} e^{i \pi 2 n \xi}
\end{gathered}
$$

There exist the following relations connecting different elliptic functions and constants with $\theta$-functions and $\theta$-constants $\theta(0) \equiv \theta(0 \mid \tau)$ :

$$
\zeta(\xi)=\eta(\tau) \xi+\left[\log \theta_{*}(\xi)\right]^{\prime}
$$

where

with

$$
\begin{gathered}
\eta(\tau)=-\frac{1}{6} \frac{\theta_{*}^{\prime \prime \prime}(0)}{\theta_{*}^{\prime}(0)} \\
\wp(\xi)=-\zeta^{\prime}(\xi)=e_{3}+\left[\frac{\theta_{*}^{\prime}(0)}{\theta_{4}(0)} \frac{\theta_{4}(\xi)}{\theta_{*}(\xi)}\right]^{2}
\end{gathered}
$$

$$
e_{3}(\tau)=-\frac{\pi^{2}}{3}\left[\theta_{2}^{4}(0)+\theta_{3}^{4}(0)\right]
$$

To simplify some formulas, we also introduce the logarithmic derivative of the $\theta$-function

$$
\bar{\zeta}(\xi \mid \tau) \equiv \zeta(\xi \mid \tau)-\eta(\tau) \xi=\left[\log \theta_{*}(\xi)\right]^{\prime}
$$

In sect.4, we also use the identity for $\theta$-functions [27]

$$
\theta_{*}(x+y) \theta_{*}(x-y) \theta_{4}^{2}(0)=\theta_{*}^{2}(x) \theta_{4}^{2}(y)-\theta_{4}^{2}(x) \theta_{*}^{2}(y)
$$

\section{Acknowledgements}

We are indebted to A.Gorsky, S.Kharchev, A.Zabrodin and especially to A.Morozov for illuminating discussions. A.Mar. is also grateful to J.Ambjorn and other members of the Theoretical High-Energy group of the Niels Bohr Institute where this work was completed, and A.Mir. acknowledges the University Roma I for warm hospitality. The work of A.Mar. was partially supported by RFBR grant 96-01-01106, INTAS grant 96-518 and the Niels Bohr Institute, the work of A.Mir. - by the grant RFBR-96-02-16347(a), the program for support of the scientific schools 96-15-96798 and INTAS-96482.

\section{References}

[1] N.Seiberg and E.Witten, "Electric-Magnetic Duality, Monopole Condensation, And Confinement in $N=2$ Supersymmetric Yang-Mills Theory ", Nucl.Phys. B426 (1994) 19-52; Erratum-ibid. B430 (1994) 485-486; hep-th/9407087

"Monopoles, Duality and Chiral Symmetry Breaking in N=2 Supersymmetric QCD", Nucl.Phys. B431 (1994) 484-550; hep-th/9408099 
[2] A.Gorsky, I.Krichever, A.Marshakov, A.Mironov and A.Morozov, "Integrability and SeibergWitten Exact Solution", Phys.Lett. B355 (1995) 466-474; hep-th/9505035

[3] E.Martinec and N.Warner, "Integrable systems and supersymmetric gauge theory", Nucl.Phys. B459 (1996) 97-112; hep-th/9509161;

T.Nakatsu and K.Takasaki, "Whitham hierarchy and N=2 supersymmetric Yang-Mills theory", Mod.Phys.Lett. A11 (1996) 157-168; hep-th/9509162;

R.Donagi and E.Witten, "Supersymmetric Yang-Mills Systems And Integrable Systems", Nucl.Phys. B460 (1996) 299; hep-th/9510101;

E.Martinec, "Integrable structures in supersymmetric gauge and string theory", Phys.Lett. B367 (1996) 91-96; hep-th/9510204;

A.Gorsky and A.Marshakov, "Towards effective topological gauge theories on spectral curves", Phys.Lett. B375 (1996) 127, hep-th/9510224;

E.Martinec and N.Warner, "Integrability in $\mathrm{N}=2$ gauge theory: a proof", hep-th/9511052;

H.Itoyama and A.Morozov, "Integrability and Seiberg-Witten Theory: Curves and Periods", Nucl.Phys. B477 (1996) 855-877; hep-th/9511126; "Prepotential and the Seiberg-Witten Theory", Nucl.Phys. B491 (1997) 529-573; hep-th/9512161;

C.Ahn and S.Nam, "Integrable structure in supersymmetric gauge theories with massive hypermultiplets", Phys.Lett. B387 (1996) 304-309; hep-th/9603028;

I.Krichever and D.Phong, "On the integrable geometry of soliton equations and N=2 supersymmetric gauge theories", J.Diff.Geom. 45 (1997) 349-389; hep-th/9604199; "Symplectic forms in the theory of solitons", hep-th/9708170;

T.Nakatsu and K.Takasaki, "Isomonodromic deformations and supersymmetric gauge theories", Int.J.Mod.Phys. A11 (1996) 5505-5518; hep-th/9603069;

K.Takasaki, "Spectral Curves and Whitham Equations in Isomonodromic Problems of Schlesinger Type", solv-int/9704004; "Dual Isomonodromic Problems and Whitham Equations", solvint/9705016;

A.Cappelli, P.Valtancoli and L.Vergnano, "Isomonodromic Properties of the Seiberg-Witten Solution", hep-th/9710248.

[4] A.Marshakov, "Exact solutions to quantum field theories and integrable equations", Mod.Phys.Lett. A11 (1996) 1169; hep-th/9602005

[5] A.Gorsky, A.Marshakov, A.Mironov and A.Morozov, " $N=2$ supersymmetric QCD and integrable spin chains: rational case $N_{f}<2 N_{c}$ ", Phys.Lett. B380 (1996) 75 ; hep-th/9603140

[6] A.Gorsky, A.Marshakov, A.Mironov and A.Morozov, "A note on spectral curve for the periodic homogeneous XYZ spin chain", hep-th/9604078

[7] A.Marshakov, "From Nonperturbative Supersymmetric Quantum Gauge Theories to Integrable Systems", in Proceedings of 10th International Conference "Problems of Quantum Field Theory", Dubna 1996, hep-th/9607159; "Non-perturbative Quantum Theories and Integrable Equations", Int.J.Mod.Phys. A12 (1997) 1607-1650; hep-th/9610242; "On Integrable Systems and Supersymmetric Gauge Theories", Theor.\& Math. Phys., July 1997; hep-th/9702083

[8] N.Nekrasov, "Five dimensional gauge theories and relativistic integrable systems", hepth/9609219

[9] A.Marshakov, A.Mironov and A.Morozov, "WDVV-like equations in N=2 SUSY Yang-Mills Theory", Phys.Lett. B389 (1996) 43-52; hep-th/9607109; 
A.Marshakov, A.Mironov and A.Morozov, "WDVV Equations from Algebra of Forms", Mod.Phys.Lett. A12 (1997) 773-788; hep-th/9701014

[10] A.Marshakov, A.Mironov and A.Morozov, "More evidences for the WDVV equations in $\mathrm{N}=2$ SUSY Yang-Mills theory", hep-th/9701123;

A.Mironov, "WDVV equations in Seiberg-Witten theory and associative algebras", hepth/9704205

[11] A. Gorsky, "Branes and integrability in the N=2 SUSY YM theory", hep-th/9612238

[12] E.Witten, "Solution of N=2 supersymmetric theories via M theory", hep-th/9703166

[13] R.Donagi, "Seiberg-Witten integrable systems", alg-geom/9705010

[14] A.Marshakov, M.Martellini and A.Morozov, "Insights and Puzzles from Branes: 4d SUSY YangMills from 6d Models", hep-th/9706050;

A. Marshakov, "Seiberg-Witten Theory, Integrable Systems and D-branes", hep-th/9709001

[15] A.Gorsky, S.Gukov and A.Mironov, " $\mathrm{N}=2$ supersymmetric field theories, integrable systems and their stringy/brane origin -I", hep-th/9707120;

A.Gorsky, S.Gukov and A.Mironov, "SUSY field theories, integrable systems and their stringy/brane origin -II", hep-th/9710239

[16] B.Dubrovin, I.Krichever and S.Novikov, in Sovremennye problemy matematiki (VINITI), Dynamical systems - 4 (1985) 179

[17] L.Faddeev and L.Takhtadjan, Hamiltonian Approach to the Theory of Solitons, 1986

[18] E.Sklyanin, Func.Anal \& Apps. 16 (1982) 27

E.Sklyanin, Func.Anal \& Apps. 17 (1983) 34

[19] A.Hanany and Y.Oz, "On the Quantum Moduli Space of Vacua of N=2 Supersymmetric $S U\left(N_{c}\right)$ Gauge Theories", Nucl. Phys. B452 (1995) 283; hep-th/9505075;

P.Argyres, M.Plesser and A.Shapere, "The Coulomb Phase of N=2 Supersymmetric QCD", Phys.Rev.Lett. 75 (1995) 1699-1702; hep-th/9505100;

J.Minahan and D.Nemeschansky, "Hyperelliptic curves for Supersymmetric Yang-Mills", Nucl.Phys. B464 (1996) 3-17; hep-th/9507032;

P.Argyres and A.Shapere, "The Vacuum Structure of N=2 SuperQCD with Classical Gauge Groups", Nucl.Phys. B461 (1996) 437-459; hep-th/9509175;

A.Hanany, "On the Quantum Moduli Space of N=2 Supersymmetric Gauge Theories", Nucl.Phys. B466 (1996) 85-100; hep-th/9509176

[20] A. Brandhuber, N. Itzhaki, V. Kaplunovsky, J. Sonnenschein and S. Yankielowicz, "Comments on the M Theory Approach to N=1 SQCD and Brane Dynamics", Phys.Lett. B410 (1997) 27-35; hep-th/9706127

[21] A.Kundu, "Generation of a quantum integrable class of discrete time or relativistic periodic Toda chains", hep-th/9403001

S.Kharchev, "Twisted systems of the $X X Z$ type", preprint ITEP, 1996; unpublished

[22] A.Brandhuber, N.Itzhaki, J.Sonnenschein, S.Theisen and S.Yankielowicz, "On the M theory approach to (compactified) $5 d$ field theories", hep-th/9709010 
[23] N.Seiberg, "Five-dimensional SUSY field theories, nontrivial fixed points, and string dynamics", Phys.Lett. B388 753; hep-th/9608111

K.Intriligator, D.R.Morrison and N.Seiberg, "Five-dimensional supersymmetric gauge theories and degenerations of Calabi-Yau Spaces", Nucl.Phys. B497 (1997) 56; hep-th/9702198

N.Seiberg and D.Morrison, "Extremal transitions and five dimensional supersymmetric fields theories", Nucl.Phys. B483 (1997) 229; hep-th/9609070

[24] A.Beilinson and A.Levin, "Elliptic polylogarithms", Proceedings of Symposia in Pure Mathematics, Vol.55, Part 2 (1994) 126-196

V.Kuznetsov, F.Nijhoff and E.Sklyanin, "Separation of variables for the Ruijsenaars system", hep-th/9701004

J.Harvey and G.Moore, "Algebra, BPS states, and strings", Nucl.Phys. B463 (1996) 315-368; hep-th/9510182

[25] I.Brunner and A.Karch, "Branes and six dimensional fixed points", hep-th/9705022

U.H.Daniellson, G.Ferretti, J.Kalkkinen and P.Stjernberg, "Notes on Supersymmetric Gauge Theories in Five and Six Dimensions", Phys.Lett. B405 (1997) 265-270; hep-th/9703098

O.Ganor, N.Seiberg and D.Morrison "Branes, Calabi-Yau spaces, and toroidal compactifications of the $N=1$ six dimensional $E_{8}$ theory", Nucl.Phys. B487 (1996) 93; hep-th/9610198

E.Witten, "New "gauge" theories" in six dimensions", hep-th/9710065

B.Kol, "On 6d "gauge" theories with irrational theta angle", hep-th/9711017

[26] N.Nekrasov and A.Lowrence, "Instanton sums and five dimensional gauge theories", hepth/9706025

[27] E.T.Whittaker and G.N.Watson, A course of modern analysis (1927), 4th ed., Cambridge, 1962 